\title{
ANOTHER EXTENSION OF ORLICZ-SOBOLEV SPACES TO METRIC SPACES
}

\author{
NOUREDDINE AÏSSAOUI
}

Received 12 May 2003

We propose another extension of Orlicz-Sobolev spaces to metric spaces based on the concepts of the $\Phi$-modulus and $\Phi$-capacity. The resulting space $N_{\Phi}^{1}$ is a Banach space. The relationship between $N_{\Phi}^{1}$ and $M_{\Phi}^{1}$ (the first extension defined in Aïssaoui (2002)) is studied. We also explore and compare different definitions of capacities and give a criterion under which $N_{\Phi}^{1}$ is strictly smaller than the Orlicz space $\mathbf{L}_{\Phi}$.

\section{Introduction}

In [22], Shanmugalingam studies extensively an extension of Sobolev spaces on metric spaces different from the approach of Hajłasz in [12]. In particular, he gives a comparison between the obtained two spaces. See also $[6,9,13,22]$ for further developments of these two theories.

Since a first extension of Orlicz-Sobolev spaces on metric spaces, denoted by $M_{\Phi}^{1}(X)$, following Hajłasz' method, was studied in [4], it is natural to examine Shanmugalingam's definition based on the notions of modulus of paths families and on the capacity. The resulting space $N_{\Phi}^{1}(X)$ is a Banach space for any $\mathcal{N}$-function $\Phi$ and the space $M_{\Phi}^{1}(X)$ continuously embeds on $N_{\Phi}^{1}(X)$ when $\Phi$ satisfies the $\Delta_{2}$ condition. We know that Lipschitz functions are dense in $M_{\Phi}^{1}(X)$ for $\Phi$ verifying the $\Delta_{2}$ condition. To expect the same result with the vaster space $N_{\Phi}^{1}(X)$, we must add some assumptions, as in the Sobolev case, on the metric space $X$, namely, $X$ must be doubling and support a $(1, \Phi)$-Poincaré inequality, and $\Phi$ verifies the $\Delta^{\prime}$ condition. Remark that when $\Phi(x)=(1 / p) x^{p}(p>1)$, we rediscover the same result in the setting of Sobolev spaces. On the other hand, when $\Omega$ is a domain in $\mathbb{R}^{N}$, we give a new characterization of the Orlicz-Sobolev space $W^{1} \mathbf{L}_{\Phi}(\Omega)$, and we show that $N_{\Phi}^{1}(\Omega)=W^{1} \mathbf{L}_{\Phi}(\Omega)$ when $\Phi$ satisfies the $\Delta_{2}$ condition. Hence, for reflexive Orlicz spaces $\mathbf{L}_{\Phi}\left(\mathbb{R}^{N}\right)$, we get $N_{\Phi}^{1}\left(\mathbb{R}^{N}\right)=M_{\Phi}^{1}\left(\mathbb{R}^{N}\right)=W^{1} \mathbf{L}_{\Phi}\left(\mathbb{R}^{N}\right)$, since we know that $M_{\Phi}^{1}\left(\mathbb{R}^{N}\right)=W^{1} \mathbf{L}_{\Phi}\left(\mathbb{R}^{N}\right)$. See [4, Theorem 3.3]. We also study the mean equivalent class with respect to $\Phi\left(\mathrm{MEC}_{\Phi}\right)$ criterion under which $N_{\Phi}^{1}(X)$ is strictly included in the Orlicz space $\mathbf{L}_{\Phi}(X)$ and we compare between natural capacities defined on $N_{\Phi}^{1}(X)$. We expect that other developments will be done in forthcoming papers. 
We organize this paper as follows. In Section 2, we list the required prerequisites from the Orlicz theory. Section 3 is reserved to the study of $\Phi$-modulus, the capacity, and Orlicz-Sobolev space $N_{\Phi}^{1}(X)$. Section 4 deals with comparison between $N_{\Phi}^{1}(X)$ and $M_{\Phi}^{1}(X)$ and with some properties of $N_{\Phi}^{1}(X)$. In Section 5, we study the $\mathrm{MEC}_{\Phi}$ criterion and we compare between some capacities.

\section{Preliminaries}

An $\mathcal{N}$-function is a continuous convex and even function $\Phi$ defined on $\mathbb{R}$, verifying $\Phi(t)>$ 0 for $t>0, \lim _{t \rightarrow 0}(\Phi(t) / t)=0$, and $\lim _{t \rightarrow+\infty}(\Phi(t) / t)=+\infty$.

We have the representation $\Phi(t)=\int_{0}^{|t|} \varphi(x) d \mathfrak{L}(x)$, where $\varphi: \mathbb{R}^{+} \rightarrow \mathbb{R}^{+}$is nondecreasing, right continuous, with $\varphi(0)=0, \varphi(t)>0$ for $t>0, \lim _{t \rightarrow 0^{+}} \varphi(t)=0$, and $\lim _{t \rightarrow+\infty} \varphi(t)=$ $+\infty$. Here $\mathfrak{L}$ stands for the Lebesgue measure. We put in the sequel, as usual, $d x=d \mathfrak{L}(x)$.

The $\mathcal{N}$-function $\Phi^{*}$ conjugate to $\Phi$ is defined by $\Phi^{*}(t)=\int_{0}^{|t|} \varphi^{*}(x) d x$, where $\varphi^{*}$ is given by $\varphi^{*}(s)=\sup \{t: \varphi(t) \leq s\}$.

Let $(X, \Gamma, \mu)$ be a measure space and $\Phi$ an $\mathcal{N}$-function. The Orlicz class $\mathscr{L}_{\Phi, \mu}(X)$ is defined by

$$
\mathscr{L}_{\Phi, \mu}(X)=\left\{f: X \longrightarrow \mathbb{R} \text { measurable }: \int_{X} \Phi(f(x)) d \mu(x)<\infty\right\}
$$

We define the Orlicz space $\mathbf{L}_{\Phi, \mu}(X)$ by

$$
\mathbf{L}_{\Phi, \mu}(X)=\left\{f: X \longrightarrow \mathbb{R} \text { measurable }: \int_{X} \Phi(\alpha f(x)) d \mu(x)<\infty \text { for some } \alpha>0\right\} .
$$

The Orlicz space $\mathbf{L}_{\Phi, \mu}(X)$ is a Banach space with the following norm, called the Luxemburg norm:

$$
|\|f\||_{\Phi, \mu, X}=\inf \left\{r>0: \int_{X} \Phi\left(\frac{f(x)}{r}\right) d \mu(x) \leq 1\right\} .
$$

If there is no confusion, we set $|\|f\||_{\Phi}=|\|f\||_{\Phi, \mu, X}$.

The Hölder inequality extends to Orlicz spaces as follows: if $f \in \mathbf{L}_{\Phi, \mu}(X)$ and $g \in$ $\mathbf{L}_{\Phi^{*}, \mu}(X)$, then $f g \in \mathbf{L}^{1}$ and

$$
\int_{X}|f g| d \mu \leq 2|\|f\||_{\Phi, \mu, X} \cdot|\|g\||_{\Phi^{*}, \mu, X}
$$

Let $\Phi$ be an $\mathcal{N}$-function. We say that $\Phi$ verifies the $\Delta_{2}$ condition if there is a constant $C>0$ such that $\Phi(2 t) \leq C \Phi(t)$ for all $t \geq 0$.

The $\Delta_{2}$ condition for $\Phi$ can be formulated in the following equivalent way: for every $C>0$, there exists $C^{\prime}>0$ such that $\Phi(C t) \leq C^{\prime} \Phi(t)$ for all $t \geq 0$.

We have always $\mathscr{L}_{\Phi, \mu}(X) \subset \mathbf{L}_{\Phi, \mu}(X)$. The equality $\mathscr{L}_{\Phi, \mu}(X)=\mathbf{L}_{\Phi, \mu}(X)$ occurs if $\Phi$ verifies the $\Delta_{2}$ condition.

We know that $\mathbf{L}_{\Phi, \mu}(X)$ is reflexive if $\Phi$ and $\Phi^{*}$ verify the $\Delta_{2}$ condition.

Note that if $\Phi$ verifies the $\Delta_{2}$ condition, then $\int \Phi\left(f_{i}(x)\right) d \mu \rightarrow 0$ as $i \rightarrow \infty$ if and only if $\left.\left\|f_{i}\right\|\right|_{\Phi, \mu, X} \rightarrow 0$ as $i \rightarrow \infty$. 
Recall that an $\mathcal{N}$-function $\Phi$ satisfies the $\Delta^{\prime}$ condition if there is a positive constant $C$ such that for all $x, y \geq 0, \Phi(x y) \leq C \Phi(x) \Phi(y)$. See [16, 21]. If an $\mathcal{N}$-function $\Phi$ satisfies the $\Delta^{\prime}$ condition, then it satisfies also the $\Delta_{2}$ condition.

Let $\Omega$ be an open set in $\mathbb{R}^{N}$, let $\mathbf{C}^{\infty}(\Omega)$ be the space of functions which, together with all their partial derivatives of any order, are continuous on $\Omega$, and let $\mathbf{C}_{0}^{\infty}\left(\mathbb{R}^{N}\right)=\mathbf{C}_{0}^{\infty}$ stand for all functions in $\mathbf{C}^{\infty}\left(\mathbb{R}^{N}\right)$ which have compact support in $\mathbb{R}^{N}$. The space $\mathbf{C}^{k}(\Omega)$ stands for the space of functions having all derivatives of order $\leq k$ continuous on $\Omega$, and $\mathbf{C}(\Omega)$ is the space of continuous functions on $\Omega$.

The (weak) partial derivative of $f$ of order $|\beta|$ is denoted by

$$
D^{\beta} f=\frac{\partial^{|\beta|}}{\partial x_{1}^{\beta_{1}} \cdot \partial x_{2}^{\beta_{2}} \cdots \partial x_{N}^{\beta_{N}}} f .
$$

Let $\Phi$ be an $\mathcal{N}$-function and $m \in \mathbb{N}$. We say that a function $f: \mathbb{R}^{N} \rightarrow \mathbb{R}$ has a distributional (weak partial) derivative of order $m$, denoted by $D^{\beta} f,|\beta|=m$, if

$$
\int f D^{\beta} \theta d x=(-1)^{|\beta|} \int\left(D^{\beta} f\right) \theta d x, \quad \forall \theta \in \mathbf{C}_{0}^{\infty} .
$$

Let $\Omega$ be an open set in $\mathbb{R}^{N}$ and denote $\mathbf{L}_{\Phi, \mathfrak{L}}(\Omega)$ by $\mathbf{L}_{\Phi}(\Omega)$. The Orlicz-Sobolev space $W^{m} \mathbf{L}_{\Phi}(\Omega)$ is the space of real functions $f$ such that $f$ and its distributional derivatives up to the order $m$ are in $\mathbf{L}_{\Phi}(\Omega)$.

The space $W^{m} \mathbf{L}_{\Phi}(\Omega)$ is a Banach space equipped with the norm

$$
|\|f\||_{m, \Phi, \Omega}=\sum_{0 \leq|\beta| \leq m}\left|\left\|D^{\beta} f\right\|\right|_{\Phi}, \quad f \in W^{m} \mathbf{L}_{\Phi}(\Omega),
$$

where $\left|\left\|D^{\beta} f\right\|\right|_{\Phi}=\left|\left\|D^{\beta} f\right\|\right|_{\Phi, \mathfrak{L}, \Omega}$.

Recall that if $\Phi$ verifies the $\Delta_{2}$ condition, then $\mathbf{C}^{\infty}(\Omega) \cap W^{m} \mathbf{L}_{\Phi}(\Omega)$ is dense in $W^{m} \mathbf{L}_{\Phi}(\Omega)$, and $\mathbf{C}_{0}^{\infty}\left(\mathbb{R}^{N}\right)$ is dense in $W^{m} \mathbf{L}_{\Phi}\left(\mathbb{R}^{N}\right)$.

For more details on the theory of Orlicz spaces, see $[1,16,17,18,21]$.

In this paper, the letter $C$ will denote various constants which may differ from one formula to the next one even within a single string of estimates.

\section{The Orlicz-Sobolev space $N_{\Phi}^{1}(X)$}

3.1. $\Phi$-modulus in metric spaces. Let $(X, d, \mu)$ be a metric, Borel measure space, such that $\mu$ is positive and finite on balls in $X$.

If $I$ is an interval in $\mathbb{R}$, a path in $X$ is a continuous map $\gamma: I \rightarrow X$. By abuse of language, the image $\gamma(I)=:|\gamma|$ is also called a path. If $I=[a, b]$ is a closed interval, then the length of a path $\gamma: I \rightarrow X$ is

$$
l(\gamma)=\operatorname{length}(\gamma)=\sup \sum_{i=1}^{n}\left|\gamma\left(t_{i+1}\right)-\gamma\left(t_{i}\right)\right|
$$


where the supremum is taken over all finite sequences $a=t_{1} \leq t_{2} \leq \cdots \leq t_{n} \leq t_{n+1}=b$. If $I$ is not closed, we set

$$
l(\gamma)=\sup l\left(\left.\gamma\right|_{J}\right)
$$

where the supremum is taken over all closed subintervals $J$ of $I$. A path is said to be rectifiable if its length is a finite number. A path $\gamma: I \rightarrow X$ is locally rectifiable if its restriction to each closed subinterval of $I$ is rectifiable.

For any rectifiable path $\gamma$, there are its associated length function $s_{\gamma}: I \rightarrow[0, l(\gamma)]$ and a unique 1-Lipschitz continuous map $\gamma_{s}:[0, l(\gamma)] \rightarrow X$ such that $\gamma=\gamma_{s} \circ s_{\gamma}$. The path $\gamma_{s}$ is the arc-length parametrization of $\gamma$.

Let $\gamma$ be a rectifiable path in $X$. The line integral over $\gamma$ of each nonnegative Borel function $\rho: X \rightarrow[0, \infty]$ is

$$
\int_{\gamma} \rho d s=\int_{0}^{l(\gamma)} \rho \circ \gamma_{s}(t) d t .
$$

If the path $\gamma$ is only locally rectifiable, we set

$$
\int_{\gamma} \rho d s=\sup \int_{\gamma^{\prime}} \rho d s,
$$

where the supremum is taken over all rectifiable subpaths $\gamma^{\prime}$ of $\gamma$. See [14] for more details.

Denote by $\Gamma_{\text {rect }}$ the collection of all nonconstant compact (i.e., $I$ is compact) rectifiable paths in $X$. If $A$ is a subset of $X$, then $\Gamma_{A}$ is the family of all paths in $\Gamma_{\text {rect }}$ that intersect the set $A$, and $\Gamma_{A}^{+}$is the family of all paths $\gamma$ in $\Gamma_{\text {rect }}$ such that the Hausdorff one-dimensional measure $\mathscr{H}_{1}(|\gamma| \cap A)$ is positive.

Definition 3.1. Let $\Phi$ be an $\mathcal{N}$-function and $\Gamma$ be a collection of paths in $X$. The $\Phi$ modulus of the family $\Gamma$, denoted by $\operatorname{Mod}_{\Phi}(\Gamma)$, is defined as

$$
\inf _{\rho \in \mathscr{F}(\Gamma)}|\|\rho\||_{\Phi},
$$

where $\mathscr{F}(\Gamma)$ is the set of all nonnegative Borel functions $\rho$ such that $\int_{\gamma} \rho d s \geq 1$ for all rectifiable paths $\gamma$ in $\Gamma$. Such functions $\rho$ used to define the $\Phi$-modulus of $\Gamma$ are said to be admissible for the family $\Gamma$.

From Definition 3.1, the $\Phi$-modulus of the family of all nonrectifiable paths is 0 .

We have the following important proposition.

Proposition 3.2. Let $\Phi$ be an $\mathcal{N}$-function and let $\Gamma$ be a collection of paths in $X$. Then the $\Phi$-modulus of the family $\Gamma$ is an outer measure on $\Gamma$. That is,

(1) $\operatorname{Mod}_{\Phi}(\varnothing)=0$,

(2) $\operatorname{Mod}_{\Phi}\left(\Gamma_{1}\right) \leq \operatorname{Mod}_{\Phi}\left(\Gamma_{2}\right)$ if $\Gamma_{1} \subset \Gamma_{2}$,

(3) $\operatorname{Mod}_{\Phi}\left(\bigcup_{i=1}^{\infty} \Gamma_{i}\right) \leq \sum_{i=1}^{\infty} \operatorname{Mod}_{\Phi}\left(\Gamma_{i}\right)$. 
Proof. Assertions (1) and (2) are obvious. We prove (3). We may assume that $\operatorname{Mod}_{\Phi}\left(\Gamma_{i}\right)<$ $\infty$ for all $i$. For $\varepsilon>0$, there is $\rho_{i} \in \mathscr{F}\left(\Gamma_{i}\right)$ such that

$$
\mid\left\|\rho_{i}\right\|_{\Phi} \leq \operatorname{Mod}_{\Phi}\left(\Gamma_{i}\right)+\varepsilon 2^{-i}
$$

Set $\rho=\sup _{i} \rho_{i}$ and $\Gamma=\bigcup_{i=1}^{\infty} \Gamma_{i}$. Since $\rho \geq \rho_{i}$ for all $i, \rho \in \mathscr{F}_{(}(\Gamma)$. Thus $\operatorname{Mod}_{\Phi}(\Gamma) \leq|\|\rho\||_{\Phi}$. By [5, Lemma 2], $|\|\rho\||_{\Phi} \leq \sum_{i=1}^{\infty}\left|\left\|\rho_{i}\right\|\right|_{\Phi}$. Hence,

$$
\operatorname{Mod}_{\Phi}(\Gamma) \leq \sum_{i=1}^{\infty} \operatorname{Mod}_{\Phi}\left(\Gamma_{i}\right)+\varepsilon .
$$

Since $\varepsilon$ is arbitrary, (3) is proved.

A property relevant to paths in $X$ is said to hold for $\Phi$-almost all paths if the family of rectifiable compact paths on which that property does not hold has $\Phi$-modulus zero.

For any path $\gamma \in \Gamma_{\text {rect }}$ and for distinct points $x$ and $y$ in $|\gamma|$, denote $\gamma_{x y}$ to be the subpath $\left.\gamma\right|_{\left[t_{x}, t_{y}\right]}$, where the two distinct numbers $t_{x}$ and $t_{y}$ are chosen from the domain of $\gamma$ such that $\gamma\left(t_{x}\right)=x$ and $\gamma\left(t_{y}\right)=y$. The subpath $\gamma_{x y}$ is not a well-defined notion as there can be more than one choice of the related numbers $t_{x}$ and $t_{y}$. Because of this ambiguity, any property that is required for one choice of the subpath $\gamma_{x y}$ is also required for all such choices of subpaths.

Definition 3.3. Let $\Phi$ be an $\mathcal{N}$-function and let $l(\gamma)$ denote the length of $\gamma$. A function $u$ is said to be absolutely continuous on $\Phi$-almost every curve $\left(\mathrm{ACC}_{\Phi}\right)$ if $u \circ \gamma$ is absolutely continuous on $[0, l(\gamma)]$ for $\Phi$-almost every rectifiable arc-length parametrized path $\gamma$ in $X$. If $X$ is a domain in $\mathbb{R}^{N}$, a function $u$ is said to have the absolute continuity on almost every line (ACL) property if on almost every line parallel to the coordinate axes with respect to the Hausdorff $(N-1)$-measure, the function is absolutely continuous. An ACL function therefore has directional derivatives almost everywhere. An ACL function is said to have the property $\mathrm{ACL}_{\Phi}$ if its directional derivatives are in $\mathbf{L}_{\Phi}$.

Definition 3.4. Let $u$ be a real-valued function on a metric space $X$. A nonnegative Borelmeasurable function $\rho$ is said to be an upper gradient of $u$ if for all compact rectifiable paths $\gamma$, the following inequality holds:

$$
|u(x)-u(y)| \leq \int_{\gamma} \rho d s
$$

where $x$ and $y$ are the end points of the path.

Definition 3.5. Let $\Phi$ be an $\mathcal{N}$-function and let $u$ be an arbitrary real-valued function on $X$. Let $\rho$ be a nonnegative Borel function on $X$. If there exists a family $\Gamma \subset \Gamma_{\text {rect }}$ such that $\operatorname{Mod}_{\Phi}(\Gamma)=0$ and the inequality (3.8) is true for all paths $\gamma$ in $\Gamma_{\text {rect }} \backslash \Gamma$, then $\rho$ is said to be a $\Phi$-weak upper gradient of $u$. If inequality (3.8) holds true for $\Phi$-modulus almost all paths in a set $B \subset X$, then $\rho$ is said to be a $\Phi$-weak upper gradient of $u$ on $B$. 
Lemma 3.6. Let $\Phi$ be an $\mathcal{N}$-function and let $\Gamma$ be a collection of paths in $X$. Then $\operatorname{Mod}_{\Phi}(\Gamma)=$ 0 if and only if there is a nonnegative Borel function $\rho$ on $X$ such that $\rho \in \mathbf{L}_{\Phi}$ and for all paths $\gamma \in \Gamma$,

$$
\int_{\gamma} \rho d s=\infty
$$

Proof. Suppose that $\operatorname{Mod}_{\Phi}(\Gamma)=0$. Then if $n \in \mathbb{N}^{*}$, there exists a nonnegative Borel function $\rho_{n}$ on $X$ such that $\rho_{n} \in \mathbf{L}_{\Phi}$ and $\left|\left\|\rho_{n}\right\|\right|_{\Phi} \leq 2^{-n}$. The function $\rho=\sum_{n=1}^{\infty} \rho_{n}$ is a nonnegative Borel function on $X$ and, by [5, Lemma 2], $|\|\rho\||_{\Phi} \leq \sum_{n=1}^{\infty}\left|\left\|\rho_{n}\right\|\right|_{\Phi}$, which implies that $\rho \in \mathbf{L}_{\Phi}$. It is evident that $\int_{\gamma} \rho d s=\infty$ for all paths $\gamma \in \Gamma$.

Assume that there is a nonnegative Borel function $\rho$ on $X$ such that $\rho \in \mathbf{L}_{\Phi}$ and for all paths $\gamma \in \Gamma, \int_{\gamma} \rho d s=\infty$. Then for each $n$, the function $2^{-n} \rho$ is admissible for calculating the $\Phi$-modulus of the family $\Gamma$. This implies that $\operatorname{Mod}_{\Phi}(\Gamma)=0$. The proof is complete.

Corollary 3.7. Let $\Phi$ be an $\mathcal{N}$-function and let $E \subset X$ be such that $\mu(E)=0$. Then $\operatorname{Mod}_{\Phi}\left(\Gamma_{E}^{+}\right)=0$.

Proof. Since $\infty \chi_{E}$ is an admissible function for calculating $\operatorname{Mod}_{\Phi}\left(\Gamma_{E}^{+}\right)$, the corollary follows by Lemma 3.6.

\subsection{The Orlicz-Sobolev space $N_{\Phi}^{1}(X)$}

Definition 3.8. Let $\Phi$ be an $\mathcal{N}$-function and let the set $\widetilde{N_{\Phi}^{1}}(X, d, \mu)$ be the collection of all real-valued function $u$ on $X$ such that $u \in \mathbf{L}_{\Phi}$ and $u$ has a $\Phi$-weak upper gradient in $\mathbf{L}_{\Phi}$.

We remark that $\widetilde{N_{\Phi}^{1}}$ is a vector space, since if $\alpha, \beta \in \mathbb{R}$ and $u, v \in \widetilde{N_{\Phi}^{1}}$ with respect to $\Phi$-weak upper gradients $\rho$ and $\sigma$, then $|\alpha| \rho+|\beta| \sigma$ is a $\Phi$-weak upper gradient of $\alpha \rho+\beta \sigma$.

If $u \in \widetilde{N_{\Phi}^{1}}$, we set

$$
|\|u\||_{\widetilde{N_{\Phi}^{1}}}=|\|u\||_{\Phi}+\inf _{\rho}|\|\rho\||_{\Phi}
$$

where the infimum is taken over all $\Phi$-weak upper gradient $\rho$ of $u$ such that $\rho \in \mathbf{L}_{\Phi}$.

If $u, v \in \widetilde{N_{\Phi}^{1}}$, let $u \sim v$ if $|\|u-v\||_{\widetilde{N_{\Phi}^{1}}}=0$. It can be easily seen that $\sim$ is an equivalence relation, partitioning $\widetilde{N_{\Phi}^{1}}$ into equivalence classes, which is a normed vector space under the norm defined by (3.10).

Definition 3.9. Let $\Phi$ be an $\mathcal{N}$-function. The Orlicz-Sobolev space corresponding to $\Phi$, denoted by $N_{\Phi}^{1}(X)$, is defined to be the space $\widetilde{N_{\Phi}^{1}}(X, d, \mu) / \backsim$ with the norm $|\|u\||_{N_{\Phi}^{1}}:=$ | $\left.\|u\|\right|_{\widetilde{N_{\Phi}^{1}}}$.

If $u, v \in \widetilde{N_{\Phi}^{1}}$, then it is easily verified that the functions $|u|, \min \{u, v\}, \max \{u, v\} \in \widetilde{N_{\Phi}^{1}}$, that if $\lambda \geq 0$, then $\min \{u, \lambda\} \in \widetilde{N_{\Phi}^{1}}$, and that if $\lambda \leq 0$, then $\max \{u, \lambda\} \in \widetilde{N_{\Phi}^{1}}$. Thus $N_{\Phi}^{1}(X)$ enjoys all the lattice properties in classical first-order Orlicz-Sobolev spaces. 
Lemma 3.10. Let $\Phi$ be an $\mathcal{N}$-function and $u \in \widetilde{N_{\Phi}^{1}}$. Then $u$ is $\mathrm{ACC}_{\Phi}$.

Proof. By hypothesis, $u \in \mathbf{L}_{\Phi}$ and $u$ has a $\Phi$-weak upper gradient $\rho \in \mathbf{L}_{\Phi}$. Let $\Gamma$ be the collection of all paths in $\Gamma_{\text {rect }}$ for which inequality (3.8) does not hold. $\operatorname{Then} \operatorname{Mod}_{\Phi}(\Gamma)=0$. Let $\Gamma_{1}$ be the collection of all paths in $\Gamma_{\text {rect }}$ that have a subpath in $\Gamma$. Then any admissible function used to estimate the $\Phi$-modulus of $\Gamma$ is an admissible function for $\Gamma_{1}$. Hence,

$$
\operatorname{Mod}_{\Phi}\left(\Gamma_{1}\right) \leq \operatorname{Mod}_{\Phi}(\Gamma)=0
$$

Let $\Gamma_{2}$ be the collection of all paths $\gamma$ in $\Gamma_{\text {rect }}$ such that $\int_{\gamma} \rho d s=\infty$. Since $\rho \in \mathbf{L}_{\Phi}$, then $\operatorname{Mod}_{\Phi}\left(\Gamma_{2}\right)=0$. Thus $\operatorname{Mod}_{\Phi}\left(\Gamma_{1} \cup \Gamma_{2}\right)=0$. If $\gamma$ is a path in $\Gamma_{\text {rect }} \backslash\left(\Gamma_{1} \cup \Gamma_{2}\right), \gamma$ has no subpath in $\Gamma_{1}$, and hence for all $x, y \in|\gamma|$,

$$
|u(x)-u(y)| \leq \int_{\gamma_{x y}} \rho d s<\infty
$$

Therefore, $u$ is absolutely continuous on each path $\gamma$ in $\Gamma_{\text {rect }} \backslash\left(\Gamma_{1} \cup \Gamma_{2}\right)$. The proof is complete.

Lemma 3.11. Let $\Phi$ be an $\mathcal{N}$-function and let $u \in \widetilde{N_{\Phi}^{1}}$ be such that $|\|u\||_{\Phi}=0$. Then $\operatorname{Mod}_{\Phi}(\Gamma)=0$, where

$$
\Gamma=\left\{\gamma \in \Gamma_{\text {rect }}: u(x) \neq 0 \text { for some } x \in|\gamma|\right\} .
$$

Proof. Since $|\|u\||_{\Phi}=0$, the set $S=\{x \in X: u(x) \neq 0\}$ has measure zero. Hence, $\Gamma=\Gamma_{S}$ and

$$
\Gamma=\Gamma_{S}^{+} \cup\left(\Gamma_{S} \backslash \Gamma_{S}^{+}\right)
$$

The subfamily $\Gamma_{S}^{+}$can be disregarded since

$$
\operatorname{Mod}_{\Phi}\left(\Gamma_{S}^{+}\right) \leq\left|\left\|\infty \cdot \chi_{S}\right\|\right|_{\Phi}=0
$$

where $\chi_{S}$ is the characteristic function of the set $S$. The paths $\gamma \in \Gamma_{S} \backslash \Gamma_{S}^{+}$intersect $S$ only on a set of linear measure zero, and hence, with respect to the linear measure almost everywhere on $\gamma$, the function $u$ takes on the value of zero. By the fact that $\gamma$ also intersect $S$, therefore, $u$ is not absolutely continuous on $\gamma$. By Lemma 3.10, $\operatorname{Mod}_{\Phi}\left(\Gamma_{S} \backslash \Gamma_{S}^{+}\right)=0$. Thus $\operatorname{Mod}_{\Phi}(\Gamma)=0$ and the proof is complete.

We deduce from the previous lemma the following corollary.

Corollary 3.12. Let $\Phi$ be an $\mathcal{N}$-function. If $u, v \in \widetilde{N_{\Phi}^{1}}$ are such that $|\|u-v\||_{\Phi}=0$, then $u$ and $v$ belong to the same equivalent class in $N_{\Phi}^{1}(X)$.

In the sequel, we will not distinguish between the functions in $\widetilde{N_{\Phi}^{1}}$ and their equivalence classes in $N_{\Phi}^{1}$. 
Lemma 3.13. Let $\Phi$ be an $\mathcal{N}$-function. If $\left(\rho_{i}\right)_{i \in \mathbb{N}^{*}}$ is a sequence of Borel functions in $\mathbf{L}_{\Phi}$ such that $\lim _{i \rightarrow \infty}\left|\left\|\rho_{i}\right\|\right|_{\Phi}=0$, then there exist a subsequence $\left(\rho_{i_{k}}\right)_{k \in \mathbb{N}^{*}}$ and a family $\Gamma \subset \Gamma_{\text {rect }}$ such that $\operatorname{Mod}_{\Phi}(\Gamma)=0$ and for all paths $\gamma \in \Gamma_{\text {rect }} \backslash \Gamma$,

$$
\lim _{k \rightarrow \infty} \int_{\gamma} \rho_{i_{k}} d s=0 .
$$

Proof. Let $\left(\rho_{i_{k}}\right)_{k \in \mathbb{N}^{*}}$ be a subsequence of the sequence $\left(\rho_{i}\right)_{i \in \mathbb{N}^{*}}$ such that $\left|\left\|\rho_{i_{k}}\right\|\right|_{\Phi} \leq 2^{-k}$. Then

$$
\begin{aligned}
\Gamma & =\left\{\gamma \in \Gamma_{\text {rect }}: \limsup _{k \rightarrow \infty} \int_{\gamma} \rho_{i_{k}} d s>0\right\} \\
& =\bigcup_{n \in \mathbb{N}}\left\{\gamma \in \Gamma_{\text {rect }}: \limsup _{k \rightarrow \infty} \int_{\gamma} \rho_{i_{k}} d s \geq \frac{1}{n}\right\} \\
& =\bigcup_{n \in \mathbb{N}}\left\{\gamma \in \Gamma_{\text {rect }}: \text { for infinitely many } k, \int_{\gamma} \rho_{i_{k}} d s \geq \frac{1}{2 n}\right\} .
\end{aligned}
$$

Hence, it suffices to show that for each $n \in \mathbb{N}$, the family of paths

$$
\Gamma_{n}=\left\{\gamma \in \Gamma_{\text {rect }}: \text { for infinitely many } k, \int_{\gamma} \rho_{i_{k}} d s \geq \frac{1}{2 n}\right\}
$$

is such that $\operatorname{Mod}_{\Phi}\left(\Gamma_{n}\right)=0$. For this goal, let $\rho=\sum_{k=1}^{\infty} \rho_{i_{k}}$. Then by [5, Lemma 2], $\rho \in \mathbf{L}_{\Phi}$. For all $\gamma \in \Gamma_{n}$,

$$
\int_{\gamma} \rho d s \geq \sum_{k=1}^{\infty} \int_{\gamma} \rho_{i_{k}} d s=\infty .
$$

Hence, $\operatorname{Mod}_{\Phi}\left(\Gamma_{n}\right)=0$. The proof is complete.

\subsection{The capacity $C_{\Phi}$}

Definition 3.14. Let $\Phi$ be an $\mathcal{N}$-function. For a set $E \subset X$, define $C_{\Phi}(E)$ by

$$
C_{\Phi}(E)=\inf \left\{|\|u\||_{N_{\Phi}^{1}}: u \in \mathscr{B}(E)\right\}
$$

where $\mathscr{B}(E)=\left\{u \in N_{\Phi}^{1}:\left.u\right|_{E} \geq 1\right\}$.

If $\mathscr{B}(E)=\varnothing$, we set $C_{\Phi}(E)=\infty$. Functions belonging to $\mathscr{B}(E)$ are called admissible functions for $E$.

We define a capacity as an increasing positive set function $C$ given on a $\sigma$-additive class of sets $\Gamma$, which contains compact sets and such that $C(\varnothing)=0$ and $C\left(\bigcup_{i \geq 1} X_{i}\right) \leq$ $\sum_{i \geq 1} C\left(X_{i}\right)$ for $X_{i} \in \Gamma, i=1,2, \ldots$

The set function $C$ is called outer capacity if for every $X \in \Gamma$,

$$
C(X)=\inf \{C(O): O \text { open, } X \subset O\} \text {. }
$$


We omit the proof of the following lemma, since it is an easy adaptation of the one [4, Theorem 4.3].

Lemma 3.15. Let $\Phi$ be an $\mathcal{N}$-function. The set function $C_{\Phi}$ is an outer capacity.

Lemma 3.16. Let $\Phi$ be an $\mathcal{N}$-function and let $\left(u_{i}\right)_{i}$ be a Cauchy sequence in $N_{\Phi}^{1}(X)$. Then there are a function $u$ in $N_{\Phi}^{1}(X)$ and a subsequence $\left(u_{i_{k}}\right)_{k}$ such that $\left(u_{i_{k}}\right)_{k}$ converges to $u$ in $\mathbf{L}_{\Phi}$ and pointwise $\mu$-almost everywhere.

Proof. Since $\left(u_{i}\right)_{i}$ is a Cauchy sequence in $N_{\Phi}^{1}(X)$, it is also a Cauchy sequence in $\mathbf{L}_{\Phi}$. By passing to a subsequence if necessary, there is a function $v \in \mathbf{L}_{\Phi}$ to which the subsequence converges both pointwise $\mu$-almost everywhere and in $\mathbf{L}_{\Phi}$. We choose a further subsequence, also denoted by $\left(u_{n}\right)_{n}$ for simplicity in notation, such that

$$
\begin{gathered}
\|\| u_{i}-v\|\|_{\Phi} \leq 2^{-i}, \\
u_{i} \longrightarrow v \text { pointwise } \mu \text {-a.e., } \\
\quad\left\|\left|\left\|g_{i+1, i}\right\|\right|_{\Phi} \leq 2^{-i},\right.
\end{gathered}
$$

where $g_{i, j}$ is an upper gradient of $u_{i}-u_{j}$. If $g_{1}$ is an upper gradient of $u_{1}$ such that $g_{1} \in \mathbf{L}_{\Phi}$, then $u_{2}=u_{1}+\left(u_{2}-u_{1}\right)$ has an upper gradient $g_{2}=g_{1}+g_{1,2}$.

In general, $u_{i}=u_{1}+\sum_{k=1}^{i-1}\left(u_{k+1}-u_{k}\right)$ has an upper gradient $g_{i}=g_{1}+\sum_{k=1}^{i-1} g_{k+1, k}$ such that $g_{i} \in \mathbf{L}_{\Phi}$.

For $j<i$,

$$
\|\| g_{i}-g_{j}\left\|\left.\right|_{\Phi} \leq \sum_{k=j}^{i-1} \mid\right\| g_{k+1, k}\|\|_{\Phi} \leq \sum_{k=j}^{i-1} 2^{-k} \leq 2^{-j+1} \longrightarrow 0 \quad \text { as } j \longrightarrow \infty .
$$

Hence, $\left(g_{i}\right)_{i}$ is a Cauchy sequence in $\mathbf{L}_{\Phi}$, which implies that it converges in $\mathbf{L}_{\Phi}$-norm to a nonnegative Borel function $g$. Let $u$ be a function defined by

$$
u(x)=\frac{1}{2}\left\{\limsup _{i \rightarrow \infty} u_{i}(x)+\liminf _{i \rightarrow \infty} u_{i}(x)\right\}
$$

whenever the definition makes sense. By (3.23), we get $u(x)=v(x) \mu$-almost everywhere, and hence, $u \in \mathbf{L}_{\Phi}$. Set $T=\left\{x: \limsup _{i \rightarrow \infty}\left|u_{i}(x)\right|=\infty\right\}$. The function $u$ is well defined outside of $T$. To prove that $u \in N_{\Phi}^{1}$, by Lemma 3.10, we must show that $u$ is well defined on almost all paths. To this end, we must prove that $\operatorname{Mod}_{\Phi}\left(\Gamma_{T}\right)=0$.

Let $\Gamma_{1}$ be the collection of all paths $\gamma \in \Gamma_{\text {rect }}$ such that either $\int_{\gamma} g d s=\infty$ or $\lim _{i \rightarrow \infty} \int_{\gamma} g_{i} d s$ $\neq \int_{\gamma} g d s$. By Lemma 3.13, $\operatorname{Mod}_{\Phi}\left(\Gamma_{1}\right)=0$. On the other hand, recall that $\Gamma_{T}^{+}=\left\{\gamma \in \Gamma_{\text {rect }}\right.$ : $\left.\mathscr{H}_{1}(|\gamma| \cap T)>0\right\}$. By (3.23), $\mu(T)=0$. Hence, $\operatorname{Mod}_{\Phi}\left(\Gamma_{T}^{+}\right)=0$. Therefore, $\operatorname{Mod}_{\Phi}\left(\Gamma_{1} \cup\right.$ $\left.\Gamma_{T}^{+}\right)=0$. Let $\gamma \in \Gamma_{\text {rect }} \backslash\left(\Gamma_{1} \cup \Gamma_{T}^{+}\right)$. Then, since $\gamma \notin \Gamma_{T}^{+}$, there exists a point $y \in|\gamma|$ such that $y \in T$. Since $g_{i}$ is an upper gradient of $u_{i}$, for any point $x \in|\gamma|$, we get

$$
\left|u_{i}(x)\right|-\left|u_{i}(y)\right| \leq\left|u_{i}(x)-u_{i}(y)\right| \leq \int_{\gamma} g_{i} d s
$$


Hence, $\left|u_{i}(x)\right| \leq\left|u_{i}(y)\right|+\int_{\gamma} g_{i} d s$. Since $\gamma \notin \Gamma_{1}$, we deduce that

$$
\limsup _{i \rightarrow \infty}\left|u_{i}(x)\right| \leq \limsup _{i \rightarrow \infty}\left|u_{i}(y)\right|+\int_{\gamma} g d s<\infty
$$

and hence $x \notin T$. Thus $\Gamma_{T} \subset \Gamma_{1} \cup \Gamma_{T}^{+}$. This implies that $\operatorname{Mod}_{\Phi}\left(\Gamma_{T}\right)=0$.

On the other hand, if $\gamma \in \Gamma_{\text {rect }} \backslash\left(\Gamma_{1} \cup \Gamma_{T}^{+}\right)$, denoting $x$ and $y$ as the end points of $\gamma$ and noting by the above argument that $x, y \notin T$, we get

$$
\begin{aligned}
|u(x)-u(y)| & =\frac{1}{2}\left|\limsup _{i \rightarrow \infty} u_{i}(x)-\liminf _{i \rightarrow \infty} u_{i}(y)+\liminf _{i \rightarrow \infty} u_{i}(x)-\limsup _{i \rightarrow \infty} u_{i}(y)\right| \\
& \leq \limsup _{i \rightarrow \infty}\left|u_{i}(x)-u_{i}(y)\right| \\
& \leq \lim _{i \rightarrow \infty} \int_{\gamma} g_{i} d s=\int_{\gamma} g d s .
\end{aligned}
$$

This means that $g$ is a weak upper gradient of $u$, and hence, $u \in N_{\Phi}^{1}$. The proof is complete.

Lemma 3.17. Let $\Phi$ be an $\mathcal{N}$-function. If $E \subset X$ is such that $C_{\Phi}(E)=0$, then $\operatorname{Mod}_{\Phi}\left(\Gamma_{E}\right)=0$. Proof. Since $C_{\Phi}(E)=0$, for each $i \in \mathbb{N}^{*}$, there exists a function $u_{i} \in N_{\Phi}^{1}$ such that $\left|\left\|u_{i}\right\|\right|_{N_{\Phi}^{1}} \leq 2^{-i}$ with $\left.u_{i}\right|_{E} \geq 1$. Pose $v_{n}=\sum_{i=1}^{n}\left|u_{i}\right|$. Then for each $n, v_{n} \in N_{\Phi}^{1}$ and

$$
\left|\left\|v_{n}-\left.v_{m}||\right|_{N_{\Phi}^{1}} \leq\left.\sum_{i=m+1}^{n}\left|\| u_{i}\right|\right|_{N_{\Phi}^{1}} \leq 2^{-m} \longrightarrow 0 \quad \text { as } m \longrightarrow \infty\right.\right.
$$

Hence, the sequence $\left(v_{n}\right)_{n}$ is a Cauchy sequence in $N_{\Phi}^{1}$. By Lemma 3.16, there is a function $v \in \mathbf{L}_{\Phi}$ such that $\mid\left\|v_{n}-v\right\| \|_{\Phi} \rightarrow 0$. By the construction used in Lemma 3.16 and since the sequence $\left(v_{n}(x)\right)_{n}$ is increasing outside of a set $T$ such that $\operatorname{Mod}_{\Phi}\left(\Gamma_{T}\right)=0$, we get

$$
v(x)=\lim _{n \rightarrow \infty} v_{n}(x)
$$

with $v(x)<\infty$.

If $E \backslash T \neq \varnothing$, then for arbitrary large $n$,

$$
\left.v\right|_{E \backslash T} \geq\left. v_{n}\right|_{E \backslash T}=\left.\sum_{i=1}^{n}\left|u_{i}\right|\right|_{E \backslash T} \geq n .
$$

Hence, $\left.v\right|_{E \backslash T}=\infty$, which is not possible because $x \notin T$. Therefore, $E \backslash T=\varnothing$, and hence, $\Gamma_{E} \subset \Gamma_{T}$. Thus $\operatorname{Mod}_{\Phi}\left(\Gamma_{E}\right)=0$. The proof is complete.

Corollary 3.18. Let $\Phi$ be an $\mathcal{N}$-function and let $E$ be a subset of $X$ such that $C_{\Phi}(E)=0$. If $u \in N_{\Phi}^{1}(X \backslash E)$, then there is an extension of $u$ to $E$ that is in $N_{\Phi}^{1}(X)$. Any two such extensions of $u$ to all of $X$ are in the same equivalence class of $N_{\Phi}^{1}(X)$. 
Theorem 3.19. For any $\mathcal{N}$-function $\Phi, N_{\Phi}^{1}(X)$ is a Banach space.

Proof. Let $\left(u_{i}\right)_{i \in \mathbb{N}^{*}}$ be a Cauchy sequence in $N_{\Phi}^{1}(X)$. It suffices to show that some subsequence is a convergent sequence in $N_{\Phi}^{1}(X)$. By passing to a subsequence if necessary, we can assume that

$$
||\left|u_{k}-u_{k+1}\right|||_{\Phi} \leq 2^{-2 k}
$$

and that

$$
\left.||\left|g_{i+1, i}\right|\right|_{\Phi} \leq 2^{-i}
$$

where $g_{i, j}$ is an upper gradient of $u_{i}-u_{j}$ chosen to satisfy the above inequality.

Let

$$
E_{k}=\left\{x \in X:\left|u_{k}(x)-u_{k+1}(x)\right| \geq 2^{-k}\right\}
$$

Then $2^{k}\left|u_{k}-u_{k+1}\right| \in N_{\Phi}^{1}(X)$ and $\left.2^{k}\left|u_{k}-u_{k+1}\right|\right|_{E_{k}} \geq 1$. Hence, by (3.33),

$$
C_{\Phi}\left(E_{k}\right) \leq 2^{k}\left|\left\|u_{k}-u_{k+1}\right\|\right|_{\Phi} \leq 2^{-k}
$$

Let $F_{j}=\cup_{k=j}^{\infty} E_{k}$ and $F=\cap_{j \in \mathbb{N}} F_{j}$. Then

$$
C_{\Phi}\left(F_{j}\right) \leq \sum_{k=j}^{\infty} C_{\Phi}\left(E_{k}\right) \leq 2^{-j+1}
$$

This implies that $C_{\Phi}(F)=0$.

For $x \in X \backslash F$, there is $j \in \mathbb{N}$ such that for all $k \in \mathbb{N}$ and $k \geq j, x \notin E_{k}$. Hence, for all $k \in \mathbb{N}$ and $k \geq j,\left|u_{k}(x)-u_{k+1}(x)\right|<2^{-k}$. Therefore, whenever $l \geq k \geq j$, we get

$$
\left|u_{k}(x)-u_{l}(x)\right| \leq 2^{-k+1}
$$

Thus the sequence $\left(u_{i}(x)\right)_{i \in \mathbb{N}^{*}}$ is a Cauchy sequence in $\mathbb{R}$, and therefore is convergent to a finite number. For $x \in X \backslash F$, we let

$$
u(x)=\lim _{i \rightarrow \infty} u_{i}(x)
$$

For $x \in X \backslash F$, we have

$$
u(x)-u_{k}(x)=\sum_{n=k}^{\infty}\left(u_{n+1}(x)-u_{n}(x)\right)
$$


By Lemma 3.17, $\operatorname{Mod}_{\Phi}\left(\Gamma_{F}\right)=0$, and for each path $\gamma \in \Gamma_{\text {rect }} \backslash \Gamma_{F}$, for all points $x \in|\gamma|$, (3.40) holds. Thus $\sum_{n=k}^{\infty} g_{n+1, n}$ is a weak upper gradient of $u-u_{k}$. Therefore,

$$
\begin{aligned}
\|\| u-\left.u_{k}||\right|_{N_{\Phi}^{1}} & \leq\left|\left\|u-\left.u_{k}||\right|_{\Phi}+\left.\sum_{n=k}^{\infty}\left|\| g_{n+1, n}\right|\right|_{\Phi}\right.\right. \\
& \leq\left.\left|\| u-u_{k}\right|\right|_{\Phi}+\sum_{n=k}^{\infty} 2^{-n} \\
& \leq\left|\| u-u_{k}\right|||_{\Phi}+2^{-k+1} \longrightarrow 0 \text { as } k \longrightarrow \infty
\end{aligned}
$$

This means that the subsequence converges in the norm of $N_{\Phi}^{1}(X)$ to $u$. The proof is complete.

In particular, we have shown that if $j \in \mathbb{N}$, there is a set $F_{j}$ such that $C_{\Phi}\left(F_{j}\right) \leq 2^{-j+1}$ and the chosen subsequence converges uniformly outside of $F_{j}$. Thus we have the following corollary.

Corollary 3.20. For any $\mathcal{N}$-function $\Phi$, any Cauchy sequence $\left(u_{i}\right)_{i \in \mathbb{N} *} \subset N_{\Phi}^{1}(X)$ has a subsequence that converges pointwise outside a set of $\Phi$-capacity zero. Furthermore, the subsequence can be chosen so that there exist sets of arbitrarily small $\Phi$-capacity such that the subsequence converges uniformly in the complement of each of these sets.

The proofs of the following three lemmas are an easy adaptation of those in [22, Lemmas 2.1.5, 2.1.7, and 2.1.8] relative to $\mathrm{L}^{p}$ Lebesgue spaces. We omit these proofs.

Lemma 3.21. Let $\Phi$ be an $\mathcal{N}$-function. Let $u_{1}$ and $u_{2}$ be $\mathrm{ACC}_{\Phi}$ functions on $X$ with $\Phi$ weak upper gradients $g_{1}$ and $g_{2}$, respectively. Let $u$ be another $\mathrm{ACC}_{\Phi}$ function in $X$ such that there is an open set $O \subset X$ verifying $u=u_{1}$ on $O$ and $u=u_{2}$ on $X \backslash O$. Then $g_{1} \chi_{O}+g_{2}$ and $g_{1}+g_{2} \chi_{X \backslash O}$ are $\Phi$-weak upper gradients of $u$.

Remark that if we are in the hypotheses of the previous lemma and if $g_{2} \geq g_{1}$ almost everywhere on $O$, then $g_{2}$ is a $\Phi$-weak upper gradient of $u$; and if $g_{2} \leq g_{1}$ almost everywhere on $X \backslash O$, then $g_{1}$ is a $\Phi$-weak upper gradient of $u$.

Lemma 3.22. Let $\Phi$ be an $\mathcal{N}$-function and let $u$ be an $\mathrm{ACC}_{\Phi}$ function on $X$ such that $u=0$ $\mu$-almost everywhere on $X \backslash O$, where $O$ is an open set in $X$. If $g$ is a $\Phi$-weak upper gradient of $u$, then $g \chi_{O}$ is also a $\Phi$-weak upper gradient of $u$.

Lemma 3.23. Let $\Phi$ be an $\mathcal{N}$-function and let $u$ be an $\mathrm{ACC}_{\Phi}$ function on $X$. If $g, h \in \mathbf{L}_{\Phi}$ are two $\Phi$-weak upper gradients of $u$ and $F$ is a closed subset of $X$, then the function $v=$ $g \chi_{F}+h \chi_{X \backslash F}$ is also a $\Phi$-weak upper gradient of $u$.

3.4. A characterization of $N_{\Phi}^{1}(X)$. Next, we define another characterization of OrliczSobolev spaces on metric measure spaces using only upper gradients and bypassing the notions of moduli of path families and weak upper gradients. We show in Theorem 3.27 that this characterization gives the same space $N_{\Phi}^{1}(X)$. 
Definition 3.24. Let $\Phi$ be an $\mathcal{N}$-function. The Orlicz-Sobolev space $H_{\Phi}^{1}(X)$ is the subspace of $\mathbf{L}_{\Phi}(X)$ defined by

$$
H_{\Phi}^{1}(X)=\left\{f \in \mathbf{L}_{\Phi}(X):|f|_{1, \Phi}<\infty\right\}
$$

where

$$
|f|_{1, \Phi}=|\|f\||_{\Phi}+\inf _{\left\{g_{i}\right\}} \liminf _{i \rightarrow \infty}\left|\| g_{i}\right|||_{\Phi} .
$$

The infimum is taken over all upper gradients $g_{i}$ of the functions $f_{i}$, where the sequence $\left(f_{i}\right)_{i}$ converges to $f$ in the space $\mathbf{L}_{\Phi}(X)$.

The proof of the following lemma is an adaptation of the one of [15, Lemma 3.1].

Lemma 3.25. Let $\Phi$ be an $\mathcal{N}$-function and let $X$ be a metric measure space. If $\left(f_{i}\right)_{i}$ is a sequence of functions in $\mathbf{L}_{\Phi}(X)$ with upper gradients $\left(g_{i}\right)_{i}$ in $\mathbf{L}_{\Phi}(X)$ such that $\left(f_{i}\right)_{i}$ weakly converges to $f$ in $\mathbf{L}_{\Phi}(X)$ and $\left(g_{i}\right)_{i}$ weakly converges to $g$ in $\mathbf{L}_{\Phi}(X)$, then $g$ is a $\Phi$-weak upper gradient of $f$, and there are convex combination sequences $\tilde{f}_{i}=\sum_{k=i}^{n_{i}} \lambda_{k, i} f_{k}$ and $\tilde{g}_{i}=\sum_{k=i}^{n_{i}} \lambda_{k, i} g_{k}$ with $\sum_{k=i}^{n_{i}} \lambda_{k, i}=1, \lambda_{k, i}>0$, so that $\left(\tilde{f}_{i}\right)_{i}$ converges in $\mathbf{L}_{\Phi}(X)$ to $f$ and $\left(\tilde{g}_{i}\right)_{i}$ converges in $\mathbf{L}_{\Phi}(X)$ to $g$.

Proof. We apply Mazur's lemma to each sequence $\left(f_{i}\right)_{i=k}^{\infty}$. We can form, for each $k$, a sequence of convex combinations of $f_{i}$ that converges in the $\mathbf{L}_{\Phi}(X)$-norm to $f$. Extracting a member from each sequence of convex combinations corresponding to each $k$, a sequence $\left(\bar{f}_{k}\right)_{k}$ can be formed so that each $\bar{f}_{k}$ is a convex combination of the original sequence $\left(f_{i}\right)_{i}$, and $\left(\bar{f}_{k}\right)_{k}$ converges in the $\mathbf{L}_{\Phi}(X)$-norm to $f$. It is easy to see that the corresponding convex combination $\left(\bar{g}_{k}\right)_{k}$ of the sequence $\left(g_{i}\right)_{i}$ is a sequence of upper gradients of $\left(\bar{f}_{k}\right)_{k}$, and because of the way $\left(\bar{f}_{k}\right)_{k}$ was formed, the sequence $\left(\bar{g}_{k}\right)_{k}$ converges weakly in $\mathbf{L}_{\Phi}(X)$ to $g$. Next, repeating this process to the pair of sequences $\left(\bar{f}_{k}\right)_{k}$ and $\left(\bar{g}_{k}\right)_{k}$, we can obtain convex combination sequences $\left(\tilde{f}_{j}\right)_{j}$ and $\left(\tilde{g}_{j}\right)_{j}$ that converge in the $\mathbf{L}_{\Phi}(X)$-norm to $f$ and $g$, respectively with $\tilde{g}_{j}$ being an upper gradient of $\tilde{f}_{j}$. The final sequences are themselves convex combinations of the original sequences, since being convex combinations of convex combinations.

A slight modification of the proof of Lemma 3.16 shows that $g$ is a $\Phi$-weak upper gradient of $f$. The proof is complete.

Corollary 3.26. Let $\Phi$ be an $\mathcal{N}$-function such that $\Phi$ and $\Phi^{*}$ satisfy the $\Delta_{2}$ condition, and let $X$ be a metric measure space equipped with a $\sigma$-finite measure. If $u$ is a function with a $\Phi$-weak upper gradient in $\mathbf{L}_{\Phi}(X)$, then there exists a $\Phi$-weak upper gradient $\rho_{u}$ in $\mathbf{L}_{\Phi}(X)$ such that if $\rho$ is another $\Phi$-weak upper gradient of $u$, then $\rho_{u} \leq \rho$ almost everywhere.

Proof. By the reflexivity of the space $\mathrm{L}_{\Phi}(X)$ and by Lemma 3.25, there is a $\Phi$-weak upper gradient $\rho_{u}$ in $\mathbf{L}_{\Phi}(X)$ with the smallest $\mathbf{L}_{\Phi}(X)$-norm among all $\Phi$-weak upper gradients in $\mathbf{L}_{\Phi}(X)$ of $u$. Let $\rho$ be another $\Phi$-weak upper gradient of $u$, and denote $E=\{x: \rho(x) \leq$ $\left.\rho_{u}(x)\right\}$. Suppose that $\mu(E)>0$. Then there is a closed subset $F$ of $E$ such that $\mu(E)>0$; see $\left[8\right.$, Theorem 2.2.2]. By Lemma 3.23, the function $\rho_{u} \chi_{X \backslash F}+\rho \chi_{F}$ is a $\Phi$-weak upper gradient of $u$, of strictly smaller $\mathbf{L}_{\Phi}(X)$-norm than $\rho_{u}$. This is impossible and hence, $\mu(E)=0$. The proof is complete. 
Theorem 3.27. Let $\Phi$ be an $\mathcal{N}$-function. Then the space $H_{\Phi}^{1}(X)$ is isometrically equivalent to the space $N_{\Phi}^{1}(X)$.

Proof. Every function in $N_{\Phi}^{1}(X)$ satisfies Definition 3.24, since we can take the sequence $\left(f_{i}\right)_{i}$ to be the function itself. By Lemma 3.25, it is clear that functions satisfying Definition 3.24 have a $\mathbf{L}_{\Phi}(X)$-representative in $N_{\Phi}^{1}(X)$. Moreover, the $N_{\Phi}^{1}(X)$-norm is equal to the norm (3.43). The proof is complete.

\section{Comparisons between $N_{\Phi}^{1}(X)$ and $M_{\Phi}^{1}(X)$ and more properties of $N_{\Phi}^{1}(X)$}

We begin by recalling the definition of the space $M_{\Phi}^{1}(X)$.

Let $u: X \rightarrow[-\infty,+\infty]$ be a $\mu$-measurable function defined on $X$. We denote by $D(u)$ the set of all $\mu$-measurable functions $g: X \rightarrow[0,+\infty]$ such that

$$
|u(x)-u(y)| \leq d(x, y)(g(x)+g(y))
$$

for every $x, y \in X \backslash F, x \neq y$ with $\mu(F)=0$. The set $F$ is called the exceptional set for $g$.

Note that the right-hand side of (4.1) is always defined for $x \neq y$. For the points $x, y \in$ $X, x \neq y$, such that the left-hand side of (4.1) is undefined, we may assume that the lefthand side is $+\infty$.

Let $\Phi$ be an $\mathcal{N}$-function. The Dirichlet-Orlicz space $\mathbf{L}_{\Phi}^{1}(X)$ is the space of all $\mu$ measurable functions $u$ such that $D(u) \cap \mathbf{L}_{\Phi}(X) \neq \varnothing$. This space is equipped with the seminorm

$$
|\|u\||_{\mathbf{L}_{\Phi}^{1}(X)}=\inf \left\{|\|g\||_{\Phi}: g \in D(u) \cap \mathbf{L}_{\Phi}(X)\right\} .
$$

The Orlicz-Sobolev space $M_{\Phi}^{1}(X)$ is defined by $M_{\Phi}^{1}(X)=\mathbf{L}_{\Phi}(X) \cap \mathbf{L}_{\Phi}^{1}(X)$ equipped with the norm

$$
|\|u\||_{M_{\Phi}^{1}(X)}=|\|u\||_{\Phi}+|\|u\||_{\mathbf{L}_{\Phi}^{1}(X)} .
$$

The following lemma is easy to verify.

Lemma 4.1. Let $\Phi$ be an $\mathcal{N}$-function and let $u \in M_{\Phi}^{1}(X)$. If $g \in D(u) \cap \mathbf{L}_{\Phi}(X)$, then there exist two functions $\tilde{u}$ and $\tilde{g}$ such that $u=\tilde{u}$ almost everywhere and $|\|g\||_{\Phi}=|\|\tilde{g}\||_{\Phi}$, and for all points $x, y \in X$,

$$
|\tilde{u}(x)-\tilde{u}(y)| \leq d(x, y)(\tilde{g}(x)+\tilde{g}(y)) .
$$

Furthermore, if $u$ is continuous in $M_{\Phi}^{1}(X)$, then it is possible to choose $u=\tilde{u}$ everywhere. Proposition 4.2. Let $\Phi$ be an $\mathcal{N}$-function. Then the set of equivalence classes of continuous function $u$ in the space $M_{\Phi}^{1}(X)$ embeds into $N_{\Phi}^{1}(X)$ with

$$
|\|u\||_{N_{\Phi}^{1}} \leq 4|\|u\||_{M_{\Phi}^{1}} .
$$

Proof. Let $u$ be a continuous representative of its equivalence class in $M_{\Phi}^{1}(X)$. Then by Lemma 4.1, for each $g^{\prime} \in D(u) \cap \mathbf{L}_{\Phi}(X)$, there is a function $g \in \mathbf{L}_{\Phi}(X)$ such that $|\|g\||_{\Phi}=$ $\left|\left\|g^{\prime}\right\|\right|_{\Phi}$, and for all points $x, y \in X,(4.1)$ holds. 
Let $x, y \in X$ and $\gamma$ be an arc-length parametrizing rectifiable path connecting $x$ to $y$. If $\int_{\gamma} g d s=\infty$, then $|u(x)-u(y)| \leq \int_{\gamma} g d s$. Suppose $\int_{\gamma} g d s<\infty$. For each $n \in \mathbb{N}$, let $\sigma_{n}$ be the partition of the domain of $\gamma$ into $n$ pieces of equal length. On each partition $\gamma_{i}=\left.\gamma\right|_{\sigma_{n}(i), \sigma_{n}(i+1)}, 0 \leq i \leq n-1$, there exists $x_{i} \in\left|\gamma_{i}\right|$ such that

$$
g\left(x_{i}\right) \leq l\left(\gamma_{i}\right)^{-1} \int_{\gamma_{i}} g d s .
$$

We note that $d\left(x_{i}, x_{i+1}\right) \leq 2 l\left(\gamma_{i}\right)$. We get

$$
\begin{aligned}
\left|u\left(x_{0}\right)-u\left(x_{n}\right)\right| & \leq \sum_{i=0}^{n-1}\left|u\left(x_{i}\right)-u\left(x_{i+1}\right)\right| \\
& \leq \sum_{i=0}^{n-1} d\left(x_{i}, x_{i+1}\right)\left(g\left(x_{i}\right)+g\left(x_{i+1}\right)\right) \\
& \leq 4 \sum_{i=0}^{n-1} \int_{\gamma_{i}} g d s=4 \int_{\gamma} g d s .
\end{aligned}
$$

Since $u$ is continuous, by letting $n \rightarrow \infty$, we obtain

$$
|u(x)-u(y)| \leq 4 \int_{\gamma} g d s
$$

Hence, the continuous representative $u$ of its equivalence class in $M_{\Phi}^{1}(X)$ belongs to an equivalence class in $N_{\Phi}^{1}(X)$ with $|\|u\||_{N_{\Phi}^{1}} \leq 4|\|u\||_{M_{\Phi}^{1}}$. By Corollary 3.12, if any representative in the equivalence class of $u$ in $M_{\Phi}^{1}(X)$ belongs to an equivalence class in $N_{\Phi}^{1}(X)$, then it belongs to the same equivalence class as $u$ in $N_{\Phi}^{1}(X)$. Thus the embedding is well defined. The proof is complete.

Theorem 4.3. Let $\Phi$ be an $\mathcal{N}$-function satisfying the $\Delta_{2}$ condition. Then the space $M_{\Phi}^{1}(X)$ continuously embeds into the space $N_{\Phi}^{1}(X)$.

Proof. The space $N_{\Phi}^{1}(X)$ is a Banach space by Theorem 3.19. Hence, by Proposition 4.2, the closure of the subspace of equivalence classes of continuous functions in $M_{\Phi}^{1}(X)$ in the norm of $M_{\Phi}^{1}(X)$ is a subspace of $N_{\Phi}^{1}(X)$. By [4, Theorem 3.10], Lipschitz functions, and therefore continuous functions, are dense in $M_{\Phi}^{1}(X)$. Thus such closure is $M_{\Phi}^{1}(X)$. The proof is complete.

Recall the following lemma in [22, Lemma 3.2.5] or [15, Lemma 2.2].

LEMMA 4.4. If $u$ is a function on $X$ such that there exist nonnegative Borel-measurable functions $g$, $h$ on $X$ with the property that

$$
|u(x)-u(y)| \leq \int_{\gamma} g d s+d(x, y)(h(x)+h(y))
$$

whenever $\gamma$ is a compact rectifiable path in $X$ with end points $x, y$, then $g+4 h$ is an upper gradient of $u$. 
Recall that if $\Phi$ and $\Phi^{*}$ satisfy the $\Delta_{2}$ condition, then $M_{\Phi}^{1}\left(\mathbb{R}^{N}\right)=W^{1} \mathbf{L}_{\Phi}\left(\mathbb{R}^{N}\right)$. See [4, Theorem 3.3]. We examine the relation between $N_{\Phi}^{1}(\Omega)$ and $W^{1} \mathbf{L}_{\Phi}(\Omega)$ when $\Omega$ is a domain in $\mathbb{R}^{N}, d(x, y)=|x-y|$, and $\mu$ is a Lebesgue $N$-measure.

We omit the proof of the following lemma, since it is exactly the same as the one in [10]; see also [23].

Lemma 4.5. Let $\Phi$ be an $\mathcal{N}$-function. If $\Omega$ is a domain in $\mathbb{R}^{N}, d(x, y)=|x-y|$, and $\mu$ is a Lebesgue $N$-measure, then $W^{1} \mathbf{L}_{\Phi}(\Omega) \subset N_{\Phi}^{1}(\Omega)$.

For the reverse inclusion, we need some auxiliary results about Orlicz and OrliczSobolev spaces on Euclidean spaces.

Let $\psi$ be a nonnegative, real-valued function in $\mathbf{C}_{0}^{\infty}\left(\mathbb{R}^{N}\right)$ such that $\operatorname{supp} \psi \subset \overline{B(0,1)}$ and $\int \psi(x) d x=1$. For $\varepsilon>0$, the function defined by $\psi_{\varepsilon}(x)=\varepsilon^{-N} \psi(x / \varepsilon)$ belongs to $\mathbf{C}_{0}^{\infty}\left(\mathbb{R}^{N}\right)$ and $\operatorname{supp} \psi_{\varepsilon} \subset \overline{B(0, \varepsilon)}$. The function $\psi_{\varepsilon}$ is called a regularizer, and the convolution $u_{\varepsilon}=$ $u * \psi_{\varepsilon}$, when it makes sense, is called the regularization of $u$. A proof of the following lemma can be deduced from [7]; see also [11]. We give a new proof inspired by [24, Theorem 1.6.1(iii)] relative to $\mathrm{L}^{p}$ Lebesgue spaces.

Lemma 4.6. Let $\Phi$ be an $\mathcal{N}$-function satisfying the $\Delta_{2}$ condition. If $u \in \mathbf{L}_{\Phi}\left(\mathbb{R}^{N}\right)$, then $u_{\varepsilon} \in$ $\mathbf{L}_{\Phi}\left(\mathbb{R}^{N}\right), \int\left(\Phi \circ u_{\varepsilon}\right)(x) d x \leq \int(\Phi \circ u)(x) d x$, and $\lim _{\varepsilon \rightarrow 0}\left|\left\|u_{\varepsilon}-u\right\|\right|_{\Phi}=0$.

Proof. By Jensen's inequality, $\left(\Phi \circ u_{\varepsilon}\right)(x) \leq(\Phi \circ u) * \psi_{\varepsilon}(x)$. Since $\Phi$ verifies the $\Delta_{2}$ condition, $\Phi \circ u \in \mathbf{L}^{1}\left(\mathbb{R}^{N}\right)$, which implies that $u_{\varepsilon} \in \mathbf{L}_{\Phi}\left(\mathbb{R}^{N}\right)$ and

$$
\int\left(\Phi \circ u_{\varepsilon}\right)(x) d x \leq \int(\Phi \circ u)(x) d x .
$$

On the other hand, for each $\delta>0$, we can find $v \in \mathbf{C}_{0}^{\infty}\left(\mathbb{R}^{N}\right)$ such that

$$
\int \Phi(|u-v|(x)) d x \leq \delta
$$

Now, since $v$ has compact support, it follows that $\int \Phi\left(\left|v_{\varepsilon}-v\right|(x)\right) d x \leq \delta$ for $\varepsilon$ sufficiently small. We apply (4.10) and (4.11) to the difference $v-u$ and obtain by using the convexity of $\Phi$ and the fact that $\Phi$ verifies the $\Delta_{2}$ condition,

$$
\begin{aligned}
\int \Phi \circ\left|u-u_{\varepsilon}\right| d x & \leq \frac{C}{3}\left[\int \Phi \circ|u-v| d x+\int \Phi \circ\left|v_{\varepsilon}-v\right| d x+\int \Phi \circ\left|v_{\varepsilon}-u_{\varepsilon}\right| d x\right] \\
& \leq C \delta .
\end{aligned}
$$

Hence, $\int \Phi \circ\left|u-u_{\varepsilon}\right| d x \rightarrow 0$ as $\varepsilon \rightarrow 0$. Since $\Phi$ verifies the $\Delta_{2}$ condition, the result follows.

Lemma 4.7. Let $\Phi$ be an $\mathcal{N}$-function satisfying the $\Delta_{2}$ condition and $u \in W^{m} \mathbf{L}_{\Phi}(\Omega)$. Then the regularizers of $u, u_{\varepsilon}$ are such that

$$
\lim _{\mathcal{\varepsilon} \rightarrow 0}\left|\left\|u_{\varepsilon}-u\right\|\right|_{m, \Phi, \Omega^{\prime}}=0
$$

whenever $\Omega^{\prime} \Subset \Omega$. When $\Omega=\mathbb{R}^{N}$, then $\lim _{\varepsilon \rightarrow 0}\left|\left\|u_{\varepsilon}-u\right\|\right|_{m, \Phi}=0$. 
Proof. Since $\Omega^{\prime}$ is a bounded domain, there is $\varepsilon_{0}>0$ such that $\varepsilon_{0}<\operatorname{dist}\left(\Omega^{\prime}, \partial \Omega\right)$. Let $\varepsilon<\varepsilon_{0}$. The differentiation under the integral in the definition of $u_{\varepsilon}$ and the application of (2.6) give for $x \in \Omega^{\prime}$ and $|\alpha| \leq m$,

$$
\begin{aligned}
D^{\alpha} u_{\varepsilon}(x) & =\varepsilon^{-N} \int_{\Omega} D_{x}^{\alpha} \psi\left(\frac{x-y}{\varepsilon}\right) u(y) d y \\
& =(-1)^{|\alpha|} \varepsilon^{-N} \int_{\Omega} D_{y}^{\alpha} \psi\left(\frac{x-y}{\varepsilon}\right) u(y) d y \\
& =\varepsilon^{-N} \int_{\Omega} \psi\left(\frac{x-y}{\varepsilon}\right) D^{\alpha} u(y) d y \\
& =\left(D^{\alpha} u\right)_{\varepsilon}(x) .
\end{aligned}
$$

The result follows from Lemma 4.6.

Corollary 4.8. Let $\Phi$ be an $\mathcal{N}$-function satisfying the $\Delta_{2}$ condition and $u \in \mathbf{L}_{\Phi}(\Omega)$. Then $u \in W^{1} \mathbf{L}_{\Phi}(\Omega)$ if and only if $u$ has a representative $\bar{u}$ that is absolutely continuous on almost all line segments in $\Omega$ parallel to the coordinate axes and whose (classical) partial derivatives belong to $\mathrm{L}_{\Phi}(\Omega)$.

Proof. Applying Lemma 4.7, we follow word by word the proof in [24, Theorem 2.14] to get the result. We omit the details.

Theorem 4.9. Let $\Phi$ be an $\mathcal{N}$-function satisfying the $\Delta_{2}$ condition. If $\Omega$ is a domain in $\mathbb{R}^{N}$, $d(x, y)=|x-y|$, and $\mu$ is a Lebesgue $N$-measure, then $W^{1} \mathbf{L}_{\Phi}(\Omega)=N_{\Phi}^{1}(\Omega)$.

Proof. By Lemma 4.5, $W^{1} \mathbf{L}_{\Phi}(\Omega) \subset N_{\Phi}^{1}(\Omega)$. It remains to prove that $N_{\Phi}^{1}(\Omega) \subset W^{1} \mathbf{L}_{\Phi}(\Omega)$. Let $u \in N_{\Phi}^{1}(\Omega)$. By Lemma 3.10, $u$ has the property ACC $_{\Phi}$ and has a $\Phi$-weak upper gradient $\rho$ in $\mathbf{L}_{\Phi}(\Omega)$. Therefore, $u$ is ACL with the principal directional gradient matrix $\nabla u$ such that by applying the fundamental theorem of calculus and a Lebesgue point argument, we easily see that $|\nabla u| \leq \rho$ almost everywhere. Hence, $u$ has the property $\mathrm{ACL}_{\Phi}$ and by Corollary 4.8, $u \in W^{1} \mathbf{L}_{\Phi}(\Omega)$. The proof is complete.

Definition 4.10. A measure $\mu$ is said to be doubling if there is a constant $C \geq 1$ such that

$$
\mu(B(x, 2 r)) \leq C \mu(B(x, r))
$$

for every $x \in X$ and $r>0$.

A metric measure space $(X, \mu, d)$ is said to be a doubling space if $\mu$ is doubling.

Recall the following result, called Poincaré inequality. See [4, Proposition 3.9].

Proposition 4.11. Let $\Phi$ be an $\mathcal{N}$-function. If $u \in M_{\Phi}^{1}(X)$ and $E \subset X$ is $\mu$-measurable with $0<\mu(E)<\infty$, then for every $g \in D(u) \cap \mathbf{L}_{\Phi}(X)$,

$$
\left|\left\|u-\left.u_{E}||\right|_{\mathbf{L}_{\Phi}(E)} \leq 2 \operatorname{diam}(E)|\|g\||_{\mathbf{L}_{\Phi}(E)},\right.\right.
$$

where $u_{E}=(1 / \mu(E)) \int_{E} f d \mu$. 
Now, we have by Hölder inequality,

$$
\int_{E}\left|u-u_{E}\right| \leq C \operatorname{diam}(E)|\|g\||_{\mathbf{L}_{\Phi}(E)}|\|1\||_{\mathbf{L}_{\Phi^{*}}(E)}
$$

Recall that $|\|1\||_{\mathbf{L}_{\Phi^{*}}(E)}=\mu(E) \Phi^{-1}(1 / \mu(X))$. This justifies the following definition.

Definition 4.12. Let $\Phi$ be an $\mathcal{N}$-function. The space $X$ is said to support a weak $(1, \Phi)$ Poincaré inequality if there are constants $C>0$ and $\tau \geq 1$ such that for all balls $B \subset X$ and all pairs of functions $u$ and $\rho$, whenever $\rho$ is an upper gradient of $u$ on $\tau B$ and $u$ is integrable on $B$, the following inequality holds:

$$
\frac{1}{\mu(B)} \int_{B}\left|u-u_{E}\right| \leq C \operatorname{diam}(B)|\|g\||_{\mathbf{L}_{\Phi}(\tau B)} \Phi^{-1}\left(\frac{1}{\mu(\tau B)}\right) .
$$

When $\tau=1$, we say that $X$ supports a $(1, \Phi)$-Poincaré inequality.

Proposition 4.13. Let $\Phi$ be an $\mathcal{N}$-function and let $X$ be a doubling space. Define the operator $M$ by

$$
M(f)(x)=\sup _{B} \frac{1}{\mu(B)} \Phi\left(|\|f\||_{\mathbf{L}_{\Phi}(B)}\right)
$$

where the supremum is taken over all balls $B \subset X$ such that $x \in B$. Then if $f \in \mathbf{L}_{\Phi}$,

$$
\lim _{\lambda \rightarrow \infty} \lambda \mu\{x \in X: M(f)(x)>\lambda\}=0 .
$$

Proof. Define for every $R>0$ the operator $M_{R}$ by

$$
\mathcal{M}_{R}(f)(x)=\sup _{B} \frac{1}{\mu(B)} \Phi\left(|\|f\||_{\mathbf{L}_{\Phi}(B)}\right)
$$

where the supremum is taken over all balls $B \subset X$ such that $x \in B$ and $\operatorname{diam}(B) \leq 2 R$. Let $f \in \mathbf{L}_{\Phi}$ and set $E_{\lambda}^{R}=\left\{x \in X: M_{R}(f)(x)>\lambda\right\}$ and $E_{\lambda}=\{x \in X: M(f)(x)>\lambda\}$. We have $E_{\lambda}^{R} \subset E_{\lambda}$. On the other hand, if $x \in E_{\lambda}^{R}$, there is $y_{x} \in X$ and $r_{x}>0$ so that $x \in B\left(y_{x}, r_{x}\right)$ and

$$
\frac{1}{\mu\left(B\left(y_{x}, r_{x}\right)\right)} \Phi\left(\mid\|f\|_{\mathbf{L}_{\Phi}\left(B\left(y_{x}, r_{x}\right)\right)}\right)>\lambda
$$

Hence, $B\left(y_{x}, r_{x}\right) \subset E_{\lambda}^{R}$. The set $E_{\lambda}^{R}$ is covered by such balls $B\left(y_{x}, r_{x}\right)$ with radii $r_{x} \leq R$. By the basic covering theorem (see, for instance, [19, Theorem 2.1 and the remark on page 23]), there exists a countable disjoint subcollection $\left\{B\left(x_{i}, r_{i}\right)\right\}_{i=1}^{\infty}$ of this covering 
collection of balls such that $E_{\lambda}^{R}$ is covered by the collection $\left\{B\left(x_{i}, 5 r_{i}\right)\right\}_{i=1}^{\infty}$. Thus

$$
\begin{aligned}
\mu\left(E_{\lambda}^{R}\right) & \leq \sum_{i=1}^{\infty} \mu\left(B\left(x_{i}, 5 r_{i}\right)\right) \\
& \leq C \sum_{i=1}^{\infty} \mu\left(B\left(x_{i}, r_{i}\right)\right) \\
& \leq C \lambda^{-1} \sum_{i=1}^{\infty} \Phi\left(|\|f\||_{\mathbf{L}_{\Phi}\left(B\left(x_{i}, r_{i}\right)\right)}\right) \\
& \leq C \lambda^{-1} \Phi\left(\sum_{i=1}^{\infty}|\|f\||_{\mathbf{L}_{\Phi}\left(B\left(x_{i}, r_{i}\right)\right)}\right) .
\end{aligned}
$$

By the same method as the one in [3, Lemma 3.2], we have

$$
\sum_{i=1}^{\infty}|\|f\||_{\mathbf{L}_{\Phi}\left(B\left(x_{i}, r_{i}\right)\right)} \leq 4|\|f\||_{\mathbf{L}_{\Phi}\left(\cup_{i} B\left(x_{i}, r_{i}\right)\right)} .
$$

Hence,

$$
\mu\left(E_{\lambda}^{R}\right) \leq C \lambda^{-1} \Phi\left(4|\|f\||_{\mathbf{L}_{\Phi}\left(\cup_{i} B\left(x_{i}, r_{i}\right)\right)}\right) \leq C \lambda^{-1} \Phi\left(4|\|f\||_{\mathbf{L}_{\Phi}\left(E_{\lambda}^{R}\right)}\right) .
$$

Thus

$$
\mu\left(E_{\lambda}^{R}\right) \leq C \lambda^{-1} \Phi\left(4|\|f\||_{\mathbf{L}_{\Phi}\left(E_{\lambda}\right)}\right) .
$$

For $0<R_{1}<R_{2}$, we have $E_{\lambda}^{R_{1}} \subset E_{\lambda}^{R_{2}}$ and $E_{\lambda}=\bigcup_{R>0} E_{\lambda}^{R}$. This implies, by taking the limit as $R \rightarrow \infty$, that

$$
\mu\left(E_{\lambda}\right) \leq C \lambda^{-1} \Phi\left(4|\|f\||_{\mathbf{L}_{\Phi}\left(E_{\lambda}\right)}\right) .
$$

Hence,

$$
\mu\left(E_{\lambda}\right) \leq C \lambda^{-1} \Phi\left(4|\|f\||_{\mathbf{L}_{\Phi}}\right),
$$

and thus $\lim _{\lambda \rightarrow \infty} \mu\left(E_{\lambda}\right)=0$. Therefore, we get $|\|f\||_{\mathbf{L}_{\Phi}\left(E_{\lambda}\right)} \rightarrow 0$ as $\lambda \rightarrow \infty$, since $f \in \mathbf{L}_{\Phi}$. By (4.27) and since $\Phi$ is continuous and increasing, we have

$$
\lim _{\lambda \rightarrow \infty} \lambda \mu\left(E_{\lambda}\right)=0 .
$$

The proof is complete.

Let $x_{0}$ be a fixed point and for each positive integer $i$, consider the following function:

$$
\eta_{i}(x)= \begin{cases}1 & \text { if } d\left(x_{0}, x\right) \leq i-1, \\ i-d\left(x_{0}, x\right) & \text { if } i-1<d\left(x_{0}, x\right)<i \\ 0 & \text { otherwise }\end{cases}
$$

It is easy to see that $\eta_{i}$ is 1 -Lipschitz. 
Lemma 4.14. Let $\Phi$ be an $\mathcal{N}$-function and let $u \in N_{\Phi}^{1}(X)$. Then the function $v_{i}=u \eta_{i}$ is also in $N_{\Phi}^{1}(X)$, and furthermore, the sequence $\left(v_{i}\right)_{i}$ converges to $u$ in $N_{\Phi}^{1}(X)$.

Proof. If $x, y \in X$, then

$$
\begin{aligned}
\left|v_{i}(x)-v_{i}(y)\right| & \leq\left|\eta_{i}(x) u(x)-\eta_{i}(x) u(y)\right|+\left|\eta_{i}(x) u(y)-\eta_{i}(y) u(y)\right| \\
& \leq|u(x)-u(y)|+|u(y)|\left|\eta_{i}(x)-\eta_{i}(y)\right| .
\end{aligned}
$$

By Lemma 4.4, if $g$ is an upper gradient of $u$, then $g+4|u|$ is also an upper gradient of $v_{i}$. Since $v_{i}$ vanishes on $X \backslash B\left(x_{0}, i\right)$, by Lemma 3.22, the function $g_{i}=(g+4|u|) \chi_{B\left(x_{0}, i\right)}$ is a $\Phi$-weak upper gradient of $v_{i}$. Since $u=v_{i}$ on $\overline{B\left(x_{0}, i-1\right)}$ and $g+g_{i}$ is a $\Phi$-weak upper gradient of $u-v_{i}$, we get by Lemma 3.22 that $\left(g+g_{i}\right) \chi_{X \backslash B\left(x_{0}, i-1\right)}$ is a $\Phi$-weak upper gradient of $u-v_{i}$. Hence,

$$
\left|\left\|u-v_{i}||_{\mathbf{L}_{\Phi}} \leq\left|\left\|u-v_{i}||_{\mathbf{L}_{\Phi}\left(X \backslash B\left(x_{0}, i-1\right)\right)} \leq 2|\|u\||_{\mathbf{L}_{\Phi}\left(X \backslash B\left(x_{0}, i-1\right)\right)} \longrightarrow 0,\right.\right.\right.\right.
$$

since $u \in \mathbf{L}_{\Phi}$. Moreover,

$$
\left.||\left|\left(g+g_{i}\right) \chi_{X \backslash B\left(x_{0}, i-1\right)}\right|\right|_{\mathbf{L}_{\Phi}} \leq 2|\|g\||_{\mathbf{L}_{\Phi}\left(X \backslash B\left(x_{0}, i-1\right)\right)}+4|\|u\||_{\mathbf{L}_{\Phi}\left(X \backslash B\left(x_{0}, i-1\right)\right)} \longrightarrow 0,
$$

since $g, u \in \mathbf{L}_{\Phi}$. Hence, $\left|\left\|u-v_{i}\right\|\right|_{N_{\Phi}^{1}} \rightarrow 0$ as $i \rightarrow \infty$. The proof is complete.

Theorem 4.15. Let $\Phi$ be an $\mathcal{N}$-function satisfying the $\Delta^{\prime}$ condition. If $X$ is a doubling space supporting a weak $(1, \Phi)$-Poincaré inequality, then Lipschitz functions are dense in $N_{\Phi}^{1}(X)$.

Proof. Let $u \in N_{\Phi}^{1}(X)$. By Lemma 4.14, we can assume that $u$ vanishes outside a bounded set. Let $g$ be an upper gradient of $u$ such that $g \in \mathbf{L}_{\Phi}(X)$ and set

$$
E_{\lambda}=\{x \in X: M(f)(x)>\Phi(\lambda)\}
$$

By Proposition 4.13, $\Phi(\lambda) \mu\left(E_{\lambda}\right) \rightarrow 0$ as $\lambda \rightarrow \infty$.

Let $x \in X \backslash E_{\lambda}$. Since $\Phi$ verifies the $\Delta^{\prime}$ condition, we get for all $r>0$,

$$
\frac{1}{\mu(B(x, r))} \int_{B(x, r)}\left|u-u_{B(x, r)}\right| \leq C r|\|g\||_{\mathbf{L}_{\Phi}(\tau B(x, r))} \Phi^{-1}\left(\frac{1}{\mu(\tau B(x, r))}\right) \leq C r \lambda
$$

Hence, for $s \in[r / 2, r]$, we get for $x \in X \backslash E_{\lambda}$,

$$
\begin{aligned}
\left|u_{B(x, s)}-u_{B(x, r)}\right| & \leq \frac{1}{\mu(B(x, s))} \int_{B(x, s)}\left|u-u_{B(x, r)}\right| \\
& \leq \frac{\mu(B(x, r))}{\mu(B(x, s))}\left[\frac{1}{\mu(B(x, r))} \int_{B(x, r)}\left|u-u_{B(x, r)}\right|\right] \\
& \leq C r \lambda .
\end{aligned}
$$

By a chaining argument, for any positive $s<r$, we get for $x \in X \backslash E_{\lambda}$,

$$
\left|u_{B(x, s)}-u_{B(x, r)}\right| \leq C r \lambda .
$$


Hence, any sequence $\left(\boldsymbol{u}_{B\left(x, r_{i}\right)}\right)_{i}$ is a Cauchy sequence in $\mathbb{R}$, and hence, is convergent. This implies that the following function is well defined on $X \backslash E_{\lambda}$ :

$$
u_{\lambda}(x):=\lim _{r \rightarrow 0} u_{B(x, r)}
$$

By [19, Theorem 2.12] or [8, Corollary 2.9.9], almost every point in $X$ is a Lebesgue point of $u$ since every function in $N_{\Phi}^{1}(X)$ is locally integrable. On the other hand, at Lebesgue points of $u$ in $X \backslash E_{\lambda}$, we have $u_{\lambda}=u$, and since $E_{\lambda}$ is open, $u-u_{\lambda}$ satisfies the hypotheses of Lemma 3.22. Now, for $x, y \in X$, consider the family of balls $\left\{B_{i}\right\}_{i=-\infty}^{\infty}$ defined by $B_{1}=B(x, d(x, y))$ and $B_{-1}=B(y, d(x, y))$ and inductively for $i>1, B_{i}=(1 / 2) B_{i-1}$ and $B_{-i}=(1 / 2) B_{-i+1}$.

For $x, y \in X \backslash E_{\lambda}$, by construction, we get

$$
\left|u_{\lambda}(x)-u_{\lambda}(y)\right| \leq \sum_{i=-\infty}^{\infty}\left|u_{B_{i}}-u_{B_{i+1}}\right| \leq C \lambda d(x, y) .
$$

Thus $u_{\lambda}$ is $C \lambda$-Lipschitz on $X \backslash E_{\lambda}$. We extend $u_{\lambda}$ as a $C \lambda$-Lipschitz extension to the entire $X$; see for instance [20]. Choose an extension such that $u_{\lambda}$ is bounded by $2 C \lambda$. This can be done by truncating any Lipschitz extension at $C \lambda$. This truncation do not affect the $u_{\lambda}$ on $X \backslash E_{\lambda}$ because for large enough $\lambda$, the original function $u$ is bounded on its Lebesgue points of $X \backslash E_{\lambda}$, since $u$ is zero outside a bounded set and hence the nonzero values of $u_{\lambda}$ in $X \backslash E_{\lambda}$ lie within a bounded set which is independent of $\lambda$, and $u_{\lambda}$ is $C \lambda$ Lipschitz.

Hence,

$$
\left|\left\|u-\left.u_{\lambda}||\right|_{\mathbf{L}_{\Phi}}=\left|\left\|u-\left.u_{\lambda}||\right|_{\mathbf{L}_{\Phi}\left(E_{\lambda}\right)} \leq|\|u\||_{\mathbf{L}_{\Phi}\left(E_{\lambda}\right)}+\left.\left|\| u_{\lambda}\right|\right|_{\mathbf{L}_{\Phi}\left(E_{\lambda}\right)} .\right.\right.\right.\right.
$$

Since $\lim _{\lambda \rightarrow \infty} \mu\left(E_{\lambda}\right)=0$, we see that $\lim _{\lambda \rightarrow \infty} \mid\|u\|_{\mathbf{L}_{\Phi}\left(E_{\lambda}\right)}=0$. Now, since $\Phi$ verifies the $\Delta^{\prime}$ condition, it satisfies also the $\Delta_{2}$ condition (see $\left.[16,21]\right)$. Hence, $\int_{E_{\lambda}} \Phi\left(u_{\lambda}\right) d \mu \leq$ $\int_{E_{\lambda}} \Phi(C \lambda) d \mu \leq C^{\prime} \Phi(\lambda) \mu\left(E_{\lambda}\right) \rightarrow 0$ as $\lambda \rightarrow \infty$. Thus $\left|\left\|u_{\lambda}\right\|\right|_{\mathbf{L}_{\Phi}\left(E_{\lambda}\right)} \rightarrow 0$ as $\lambda \rightarrow \infty$. This implies $\left.\left|\| u-u_{\lambda}\right|\right|_{\mathbf{L}_{\Phi}} \rightarrow 0$ as $\lambda \rightarrow \infty$. By Lemma 3.22, the function $(C \lambda+g) \chi_{E_{\lambda}}$ is a $\Phi$-weak upper gradient of $u-u_{\lambda}$. Hence, $u-u_{\lambda} \in N_{\Phi}^{1}(X)$ and also $u_{\lambda} \in N_{\Phi}^{1}(X)$. Since

$$
\int_{X} \Phi\left(C \lambda \chi_{E_{\lambda}}\right) d \mu \leq C^{\prime} \Phi(\lambda) \mu\left(E_{\lambda}\right) \longrightarrow 0 \quad \text { as } \lambda \longrightarrow \infty
$$

we get $\left|\left\|C \lambda \chi_{E_{\lambda}}\right\|\right|_{\mathbf{L}_{\Phi}} \rightarrow 0$ as $\lambda \rightarrow \infty$. For $g \chi_{E_{\lambda}}$, we have

$$
\|\| g \chi_{E_{\lambda}}||_{\mathbf{L}_{\Phi}}=|\|g\||_{\mathbf{L}_{\Phi}\left(E_{\lambda}\right)} \longrightarrow 0 \text { as } \lambda \longrightarrow \infty,
$$

since $g \in \mathbf{L}_{\Phi}(X)$ and $\mu\left(E_{\lambda}\right) \rightarrow 0$ as $\lambda \rightarrow \infty$. Thus the sequence $\left(u_{\lambda}\right)$ converges to $u$ in $N_{\Phi}^{1}(X)$. The proof is complete.

By Corollary 3.20 and Theorem 4.15 we obtain the following corollary.

Corollary 4.16. Let $\Phi$ be an $\mathcal{N}$-function satisfying the $\Delta^{\prime}$ condition. If $X$ is a doubling space supporting a weak $(1, \Phi)$-Poincaré inequality, then for each function $u$ in $N_{\Phi}^{1}(X)$, there are open sets of arbitrarily small $\Phi$-capacity such that $u$ is continuous in the complement of these sets. 


\section{The $\mathrm{MEC}_{\Phi}$ criterion and other capacities}

In this section, we show that $N_{\Phi}^{1}(X)$ is strictly smaller than the Orlicz space $\mathbf{L}_{\Phi}(X)$, and we give comparisons between some capacities.

Definition 5.1. Let $\Phi$ be an $\mathcal{N}$-function and let $\rho$ be a nonnegative Borel function in $X$ such that $\rho \in \mathbf{L}_{\Phi}(X)$. Define the equivalence relation $\mathscr{R}_{\rho}$ by $x \mathscr{R}_{\rho} y$, for $x, y \in X$, if either $x=y$ or there is a path $\gamma \in \Gamma_{\text {rect }}$ connecting $x$ to $y$ such that $\int_{\gamma} \gamma d s<\infty$.

It is easily seen that $\mathscr{R}_{\rho}$ is an equivalence relation partitioning $X$ into equivalent classes.

Definition 5.2. Let $\Phi$ be an $\mathcal{N}$-function. A metric measure space is said to admit the $\mathrm{MEC}_{\Phi}$ if each nonnegative Borel function $\rho$ in $X$, belonging to $\mathrm{L}_{\Phi}(X)$, generates an equivalent class $G_{\rho}$, called the main equivalence class of $\rho$, such that $\mu\left(X \backslash G_{\rho}\right)=0$.

Proposition 5.3. Let $\Phi$ be an $\mathcal{N}$-function and let $X$ be an $\mathrm{MEC}_{\Phi}$ space containing two disjoint open sets. For any subset $E \subset X$, the following equivalence holds:

$$
\operatorname{Mod}_{\Phi}\left(\Gamma_{E}\right)=0 \Longleftrightarrow C_{\Phi}(E)=0 .
$$

Proof. By Lemma 3.17, if $C_{\Phi}(E)=0$, then $\operatorname{Mod}_{\Phi}\left(\Gamma_{E}\right)=0$. Now, let $E \subset X$ be such that $\operatorname{Mod}_{\Phi}\left(\Gamma_{E}\right)=0$. Then by Lemma 3.6, there exists a nonnegative Borel function $\rho$ on $X$ belonging to $\mathbf{L}_{\Phi}(X)$ such that for all $\gamma \in \Gamma_{E}, \int_{\gamma} \rho d s=\infty$. By the $\operatorname{MEC}_{\Phi}$ property of $X, \rho$ has a main equivalence class $G_{\rho}$. Since $X$ contains two disjoint open sets and the open sets have positive measure, $G_{\rho}$ has more than one element. Let $x \in E$ and $y \in G_{\rho}$ with $y \neq x$; then any path connecting $x$ to $y$ is in $\Gamma_{E}$. Thus, by the choice of $\rho$, we get that $x$ is not equivalent to $y$ via the relation $\mathscr{R}_{\rho}$. Hence, $E$ is a subset of $X \backslash G_{\rho}$, which implies that $\mu(E)=0$. Therefore, the function $u=\chi_{E}$ is in $\mathbf{L}_{\Phi}(X)$ and is absolutely continuous on all the paths in $\Gamma_{\text {rect }}$ that are not in $\Gamma_{E}$. Since the zero function is a $\Phi$-weak upper gradient of $u, u \in N_{\Phi}^{1}(X)$. Then

$$
C_{\Phi}(E) \leq|\|u\||_{N_{\Phi}^{1}}=0
$$

The proof is complete.

In the proof of the above lemma we have shown the following proposition.

Proposition 5.4. Let $\Phi$ be an $\mathcal{N}$-function and let $X$ be an $\mathrm{MEC}_{\Phi}$ space containing two disjoint open sets. If $E \subset X$ and $\operatorname{Mod}_{\Phi}\left(\Gamma_{E}\right)=0$, then $\mu(E)=0$.

Corollary 5.5. Let $\Phi$ be an $\mathcal{N}$-function and let $X$ be an $\mathrm{MEC}_{\Phi}$ space containing two disjoint open sets. Then $\operatorname{Mod}_{\Phi}\left(\Gamma_{\text {rect }}\right)>0$.

Proof. We have $C_{\Phi}(X)>0$, since $\mu(X)>0$. Recall that $|\|1\||_{\Phi}=1 / \Phi^{-1}(1 / \mu(X))$ and hence, | $\left.\|1\|\right|_{\Phi}>0$. Now, let $u \in N_{\Phi}^{1}(X)$ and $u \geq 1$. Then

$$
|\|u\||_{N_{\Phi}^{1}} \geq|\|1\||_{\Phi}
$$

Thus

$$
C_{\Phi}(X) \geq|\|1\||_{\Phi}>0 .
$$

Proposition 5.3 implies that $\operatorname{Mod}_{\Phi}\left(\Gamma_{\text {rect }}\right)>0$. The proof is complete. 
The following proposition gives conditions on $X$ under which $N_{\Phi}^{1}(X)$ is strictly smaller than $\mathbf{L}_{\Phi}(X)$.

Proposition 5.6. Let $\Phi$ be an $\mathcal{N}$-function and let $X$ be an $\mathrm{MEC}_{\Phi}$ space containing at least two distinct points. Then there exists an equivalence class of functions in $\mathbf{L}_{\Phi}(X)$ such that no function $u$ in this equivalence class is in an equivalence class of $N_{\Phi}^{1}(X)$. In particular, $N_{\Phi}^{1}(X)$ is strictly smaller than $\mathbf{L}_{\Phi}(X)$.

Proof. Under the hypothesis, there exists an open ball $B \subset X$ such that $\mu(B)>0$ and $\mu(X \backslash B)>0$. Let $u=\chi_{B}$ and $[u]$ its equivalence class in $\mathbf{L}_{\Phi}(X)$. We know that $\left|\left\|\chi_{B}\right\|\right|_{\Phi}=$ $1 / \Phi^{-1}(1 / \mu(B))<\infty$. Suppose that $v$ is a function in this equivalence class such that $v \in$ $\widetilde{N_{\Phi}^{1}}$. Then $v(x)=1$ for almost all $x \in B$ and $v(x)=0$ for almost all $x \in X \backslash B$. Let $E=$ $\{x \in X: u(x) \neq v(x)\}$ and let $\Gamma_{v}$ be the collection of paths on which $v$ is not absolutely continuous. Hence, $\mu(E)=0$ which implies by Lemma 3.10 that $\operatorname{Mod}_{\Phi}\left(\Gamma_{E}^{+} \cup \Gamma_{v}\right)=0$. By Lemma 3.6, there is a nonnegative Borel function $\rho$ on $X$ belonging to $\mathrm{L}_{\Phi}(X)$ such that for all $\gamma \in \Gamma_{E}^{+} \cup \Gamma_{v}, \int_{\gamma} \rho d s=\infty$. Since $X$ is an MEC $_{\Phi}$ space, $\rho$ has a main equivalence class $G$ with $\mu(X \backslash G)=0$. Hence, there is $x \in B$ and $y \in X \backslash B$ so that $x, y \in G$ : there is a rectifiable path $\gamma$ connecting $x$ to $y$ so that $\int_{\gamma} \rho d s<\infty$. On the other hand, $\gamma \notin \Gamma_{E}^{+} \cup \Gamma_{v}$, and hence $v$ is absolutely continuous on $\gamma$ and

$$
\mathscr{H}_{1}(|\gamma| \cap B \cap E)=0=\mathscr{H}_{1}(|\gamma| \cap(X \backslash B) \cap E) .
$$

Let $x_{0}$ be the point in $|\gamma|$ at which $\gamma$ first leaves the closed set $X \backslash B$ (such a point exists since $|\gamma|$ is compact). The function $v$ cannot be continuous at $x_{0}$ as every neighbourhood in $|\gamma|$ of $x_{0}$ contains points at which $v$ is zero and also points at which $v$ is 1 . Thus $v \notin \widetilde{N_{\Phi}^{1}}$. This completes the proof.

Definition 5.7. Let $\Phi$ be an $\mathcal{N}$-function. For a set $E \subset X$, define $D_{\Phi}(E)$ by

$$
D_{\Phi}(E)=\inf _{u}|\|u\||_{N_{\Phi}^{1}}
$$

where the infimum is taken over all $u \in N_{\Phi}^{1}(X)$ such that for $\Phi$-almost all paths $\gamma$ intersecting $E$, the limit of $u \circ \gamma(t)$ along $\gamma$, as $\gamma(t)$ converges to any intersecting point in $E$, exists and is not smaller than 1 .

This definition in the setting of Sobolev spaces on metric spaces is used in [22], and for Sobolev spaces in the Euclidean spaces in [2].

Definition 5.8. Let $\Phi$ be an $\mathcal{N}$-function. For a set $E \subset X$, define $G_{\Phi}(E)$ by

$$
G_{\Phi}(E)=\inf \left\{|\|u\||_{N_{\Phi}^{1}}: u \in \mathscr{C}(E)\right\}
$$

where $\mathscr{C}(E)=\left\{u \in N_{\Phi}^{1}:\left.u\right|_{E} \geq 1\right.$ in a neighbourhood of $\left.E\right\}$. 
Remark 5.9. It is easily seen that if $\delta \in \mathbb{R}$ and $u \in N_{\Phi}^{1}$, then the function $v=\min \{u, \delta\} \in$ $N_{\Phi}^{1}$ with any $\Phi$-weak upper gradient of $u$ also being a $\Phi$-weak upper gradient of $v$. Hence, the condition $\left.u\right|_{E} \geq 1$ in a neighbourhood of $E$ can be replaced by the condition $\left.u\right|_{E}=1$ in a neighbourhood of $E$. By the same reasoning, it can also be assumed that these test functions are nonnegative.

Lemma 5.10. Let $\Phi$ be an $\mathcal{N}$-function. If $E \subset X$, then

$$
D_{\Phi}(E) \leq C_{\Phi}(E) \leq G_{\Phi}(E) .
$$

Proof. Let $u \in N_{\Phi}^{1}(X)$ be such that $\left.u\right|_{E} \geq 1$. Then by Lemma 3.10, $u$ is $\mathrm{ACC}_{\Phi}$ and hence it is also an admissible test function in determining $D_{\Phi}(E)$. The second inequality in (5.8) is evident. This completes the proof.

Proposition 5.11. Let $\Phi$ be an $\mathcal{N}$-function and let $X$ be such that Lipschitz functions are dense in $N_{\Phi}^{1}(X)$. If $E \subset X$, then

$$
G_{\Phi}(E)=C_{\Phi}(E)
$$

Proof. It suffices to show that $G_{\Phi}(E) \leq C_{\Phi}(E)$. Let $1>\varepsilon>0$ and let $u \in N_{\Phi}^{1}(X)$ be such that $0 \leq u \leq 1,\left.u\right|_{E}=1$, and $C_{\Phi}(E) \geq|\|u\||_{N_{\Phi}^{1}}-\varepsilon$. By Corollary 4.16, the function $u$ is $\Phi$-quasicontinuous. Thus there is an open set $F_{\varepsilon} \subset X$ such that $C_{\Phi}\left(F_{\varepsilon}\right) \leq \varepsilon$ and $u$ is continuous on $X \backslash F_{\varepsilon}$. Hence, there exists an open set $O_{\varepsilon} \subset X$ such that $\left.u\right|_{O_{\varepsilon} \backslash F_{\varepsilon}} \geq 1-\varepsilon$ and $E \subset O_{\varepsilon}$. Let $w_{\varepsilon} \in N_{\Phi}^{1}(X)$ be such that $\left.w_{\varepsilon}\right|_{O_{\varepsilon}}=1,0 \leq w_{\varepsilon} \leq 1$, and $\left|\left\|w_{\varepsilon}\right\|\right|_{N_{\Phi}^{1}} \leq 2 \varepsilon$. Define $v_{\varepsilon}$ by $v_{\varepsilon}=u /(1-\varepsilon)+w_{\varepsilon}$. Then $\left.v_{\varepsilon}\right|_{O_{\varepsilon}} \geq 1, v_{\varepsilon} \in N_{\Phi}^{1}(X)$, and

$$
G_{\Phi}(E) \leq\left|\left\|\left.v_{\varepsilon}||\right|_{N_{\Phi}^{1}} \leq \frac{1}{1-\varepsilon}|\|u\||_{N_{\Phi}^{1}}+\left|\| w_{\varepsilon}\right|||_{N_{\Phi}^{1}} .\right.\right.
$$

Hence,

$$
G_{\Phi}(E) \leq \frac{1}{1-\varepsilon}\left(C_{\Phi}(E)+\varepsilon\right)+2 \varepsilon .
$$

We get the result by letting $\varepsilon \rightarrow 0$.

Proposition 5.12. Let $\Phi$ be an $\mathcal{N}$-function. If $X$ is an $\mathrm{MEC}_{\Phi}$ space, then for all $E \subset X$,

$$
C_{\Phi}(E)=D_{\Phi}(E) .
$$

Proof. It suffices to prove that $C_{\Phi}(E) \leq D_{\Phi}(E)$. Let $u \in N_{\Phi}^{1}(X)$ be such that for $\Phi$-almost every path $\gamma \in \Gamma_{E}$,

$$
\lim _{\gamma(t) \rightarrow|\gamma| \cap E}(u \circ \gamma(t)) \geq 1 .
$$

Let $E_{1}=\{x \in E: u(x)<1\}$. Then for each $\gamma \in \Gamma_{E_{1}}$, either $\lim _{\gamma(t) \rightarrow|\gamma| \cap E_{1}}(u \circ \gamma(t))<1$ or else, either the limit does not exist or the limit exists and is greater than or equal to 1 , while $u$ is less than 1 at the limiting point in $|\gamma| \cap E_{1}$. That is, $u$ is not absolutely continuous on $\gamma$. By the choice of $u$ and by Lemma 3.10, $\operatorname{Mod}_{\Phi}\left(\Gamma_{E_{1}}\right)=0$. By Lemma 3.6 and since 
$X$ is an $\mathrm{MEC}_{\Phi}$ space, it follows that $\mu\left(E_{1}\right)=0$. Hence, the value of $u$ can be adjusted on $E_{1}$ to be greater than or equal to 1 to obtain a function in $N_{\Phi}^{1}(X)$ in the same $N_{\Phi}^{1}(X)$ equivalence class as $u$, but with the property of being greater than or equal to 1 on all of $E$. Thus $C_{\Phi}(E) \leq D_{\Phi}(E)$. The proof is complete.

Corollary 5.13. Let $\Phi$ be an $\mathcal{N}$-function and let $X$ be such that Lipschitz functions are dense in $N_{\Phi}^{1}(X)$. If $X$ is an $\mathrm{MEC}_{\Phi}$ space, then for all $E \subset X$,

$$
C_{\Phi}(E)=D_{\Phi}(E)=G_{\Phi}(E)
$$

\section{References}

[1] R. A. Adams, Sobolev Spaces, Pure and Applied Mathematics, Academic Press, New York, 1975.

[2] H. Aikawa and M. Ohtsuka, Extremal length of vector measures, Ann. Acad. Sci. Fenn. Math. 24 (1999), no. 1, 61-88.

[3] N. Aïssaoui, Capacitary type estimates in strongly nonlinear potential theory and applications, Rev. Mat. Complut. 14 (2001), no. 2, 347-370.

[4] Strongly nonlinear potential theory of metric spaces, Abstr. Appl. Anal. 7 (2002), no. 7, 357-374.

[5] N. Aïssaoui and A. Benkirane, Capacités dans les espaces d'Orlicz [Capacities in Orlicz spaces], Ann. Sci. Math. Québec 18 (1994), no. 1, 1-23 (French).

[6] J. Cheeger, Differentiability of Lipschitz functions on metric measure spaces, Geom. Funct. Anal. 9 (1999), no. 3, 428-517.

[7] T. K. Donaldson and N. S. Trudinger, Orlicz-Sobolev spaces and imbedding theorems, J. Functional Analysis 8 (1971), 52-75.

[8] H. Federer, Geometric Measure Theory, Die Grundlehren der mathematischen Wissenschaften, vol. 153, Springer-Verlag, New York, 1969.

[9] B. Franchi, P. Hajłasz, and P. Koskela, Definitions of Sobolev classes on metric spaces, Ann. Inst. Fourier (Grenoble) 49 (1999), no. 6, 1903-1924.

[10] B. Fuglede, Extremal length and functional completion, Acta Math. 98 (1957), 171-219.

[11] J.-P. Gossez, Nonlinear elliptic boundary value problems for equations with rapidly (or slowly) increasing coefficients, Trans. Amer. Math. Soc. 190 (1974), 163-205.

[12] P. Hajłasz, Sobolev spaces on an arbitrary metric space, Potential Anal. 5 (1996), no. 4, 403-415.

[13] P. Hajłasz and P. Koskela, Sobolev met Poincaré, Mem. Amer. Math. Soc. 145 (2000), no. 688, $\mathrm{x}+101$.

[14] J. Heinonen and P. Koskela, Quasiconformal maps in metric spaces with controlled geometry, Acta Math. 181 (1998), no. 1, 1-61.

[15] S. Kallunki and N. Shanmugalingam, Modulus and continuous capacity, Ann. Acad. Sci. Fenn. Math. 26 (2001), no. 2, 455-464.

[16] M. A. Krasnosel'skiǐ and J. B. Rutickiĭ, Convex Functions and Orlicz Spaces, P. Noordhoff, Groningen, 1961, translated from the first Russian edition by Leo F. Boron.

[17] A. Kufner, O. John, and S. Fučík, Function Spaces, Monographs and Textsbooks on Mechanics of Solids and Fluids, Noordhoff International Publishing, Leyden, 1977.

[18] W. A. J. Luxemburg, Banach Function Spaces, Thesis, Technische Hogeschool te Delft, 1955.

[19] P. Mattila, Geometry of Sets and Measures in Euclidean Spaces, Cambridge Studies in Advanced Mathematics, vol. 44, Cambridge University Press, Cambridge, 1995.

[20] E. J. McShane, Extension of range of functions, Bull. Amer. Math. Soc. 40 (1934), 837-842.

[21] M. M. Rao and Z. D. Ren, Theory of Orlicz Spaces, Pure and Applied Mathematics, vol. 146, Marcel Dekker, New York, 1991. 


\section{Another extension of Orlicz-Sobolev spaces to metric spaces}

[22] N. Shanmugalingam, Newtonian spaces: an extension of Sobolev spaces to metric measure spaces, Rev. Mat. Iberoamericana 16 (2000), no. 2, 243-279.

[23] J. Väisälä, Lectures on n-Dimensional Quasiconformal Mappings, Lecture Notes in Mathematics, vol. 229, Springer-Verlag, Berlin, 1971.

[24] W. P. Ziemer, Weakly Differentiable Functions, Graduate Texts in Mathematics, vol. 120, Springer-Verlag, New York, 1989.

Noureddine Aïssaoui: Département de Mathématiques, Ecole Normale Supérieure, 5206 Ben Souda, Fès, Morocco

E-mail address: n.aissaoui@caramail.com 


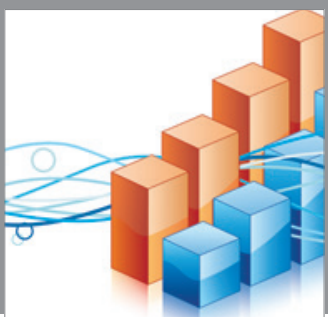

Advances in

Operations Research

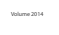

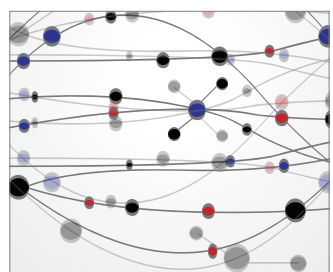

\section{The Scientific} World Journal
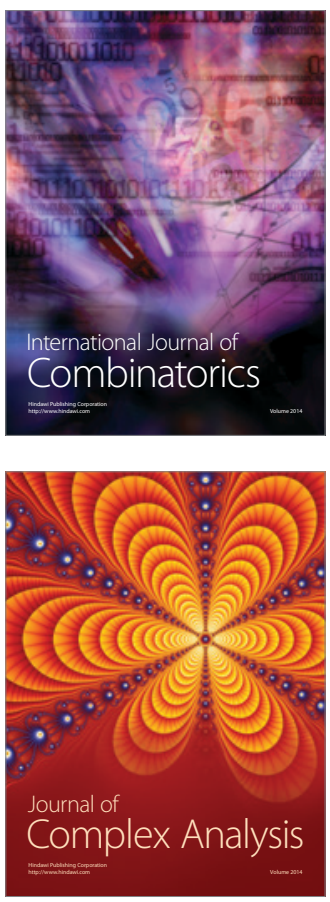

International Journal of

Mathematics and

Mathematical

Sciences
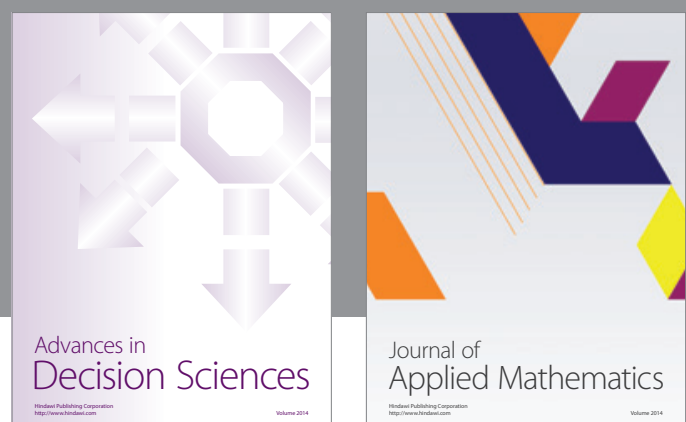

Journal of

Applied Mathematics
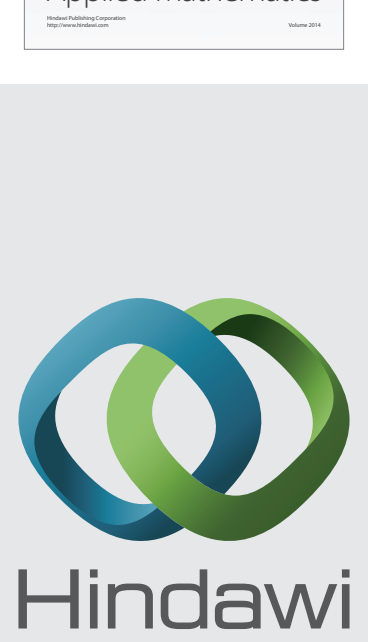

Submit your manuscripts at http://www.hindawi.com
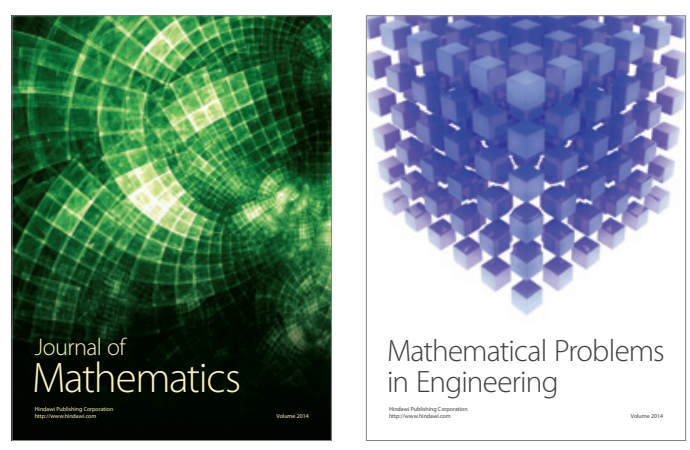

Mathematical Problems in Engineering
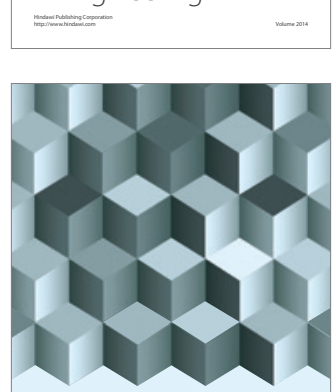

Journal of

Function Spaces
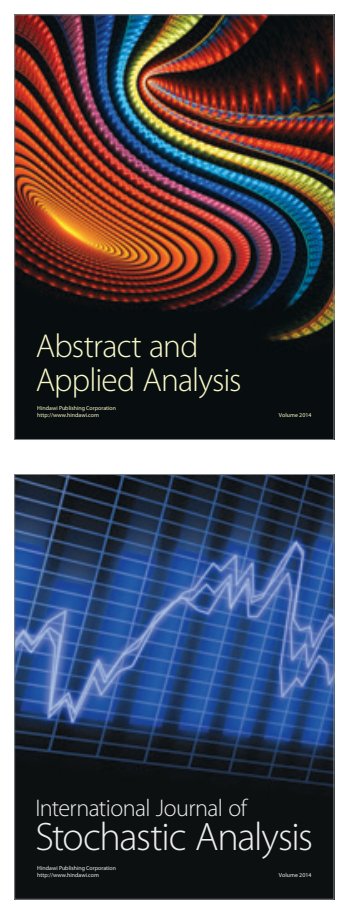

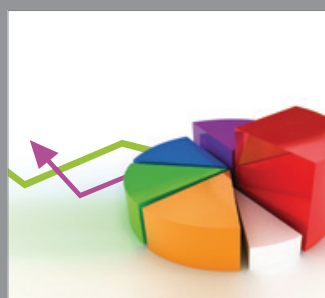

ournal of

Probability and Statistics

Promensencen
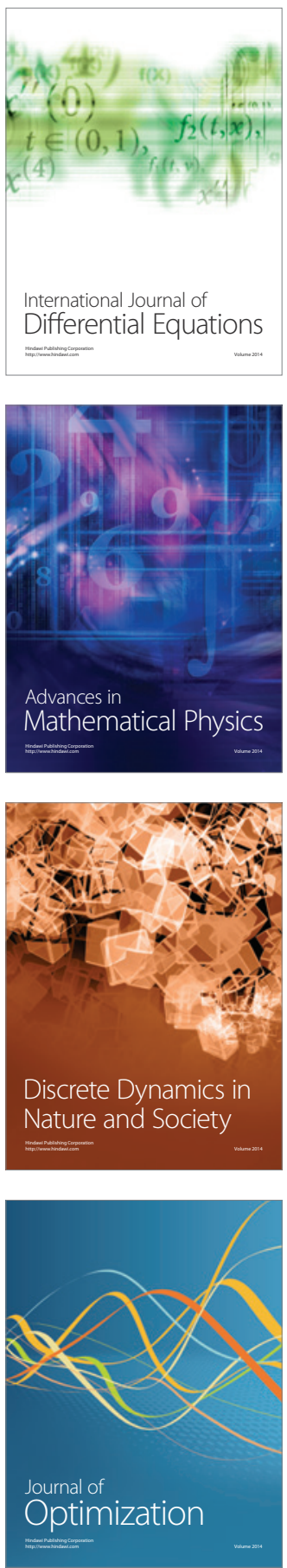